\title{
TWISTED SECTORS FOR TENSOR PRODUCT VERTEX OPERATOR ALGEBRAS ASSOCIATED TO PERMUTATION GROUPS
}

\author{
KATRINA BARRON, CHONGYING DONG, AND GEOFFREY MASON
}

\begin{abstract}
Let $V$ be a vertex operator algebra, and for $k$ a positive integer, let $g$ be a $k$-cycle permutation of the vertex operator algebra $V^{\otimes k}$. We prove that the categories of weak, weak admissible and ordinary $g$-twisted modules for the tensor product vertex operator algebra $V^{\otimes k}$ are isomorphic to the categories of weak, weak admissible and ordinary $V$-modules, respectively. The main result is an explicit construction of the weak $g$-twisted $V^{\otimes k}$-modules from weak $V$-modules. For an arbitrary permutation automorphism $g$ of $V^{\otimes k}$ the category of weak admissible $g$-twisted modules for $V^{\otimes k}$ is semisimple and the simple objects are determined if $V$ is rational. In addition, we extend these results to the more general setting of $\gamma g$-twisted $V^{\otimes k}$-modules for $\gamma$ a general automorphism of $V$ acting diagonally on $V^{\otimes k}$ and a $g$ a permutation automorphism of $V^{\otimes k}$.
\end{abstract}

\section{INTRODUCTION}

Orbifold theory and coset construction theory GKO are two important ways of constructing a new conformal field theory from a given one. The first orbifold conformal field theory was introduced in [FLM1] and the theory of orbifold conformal fields was subsequently developed, for example, in DHVW1, [DHVW2], [FLM3], and [DVVV]. The first critical step in orbifold conformal field theory is to construct twisted sectors. In [Le1] and [FLM2], twisted sectors for finite automorphisms of even lattice vertex operator algebras were first constructed - the twisted vertex operators were constructed, and in [Le2] and DL2], the twisted Jacobi identity was formulated and shown to hold for these operators. These results led to the introduction of the notion of $g$-twisted $V$-module [FFR], [D], for $V$ a vertex operator algebra and $g$ an automorphism of $V$. This notion records the properties obtained in [Le1, Section 3.3 of [FLM2], and [Le2], and provides an axiomatic definition of twisted sectors.

The focus of this paper is the construction of twisted sectors for permutation orbifold theory. Let $V$ be a vertex operator algebra, and for a fixed positive integer $k$, consider the tensor product vertex operator algebra $V^{\otimes k}$ (see [FLM3], [FHL]). Any

1991 Mathematics Subject Classification. Primary 17B68, 17B69, 17B81, 81R10, 81T40 .

Key words and phrases. Vertex operator algebras, twisted sectors, permutation orbifold, conformal field theory.

The first author was supported by an NSF Mathematical Sciences Postdoctoral Research Fellowship and a University of California President's Postdoctoral Fellowship.

The second author was supported by NSF grant DMS-9700923 and a research grant from the Committee on Research, UC Santa Cruz.

The third author was supported by NSF grant DMS-9700909 and a research grant from the Committee on Research, UC Santa Cruz. 
element $g$ of the symmetric group $S_{k}$ acts on $V^{\otimes k}$ in the obvious way, and thus it is appropriate to consider $g$-twisted $V^{\otimes k}$-modules. This is the setting for permutation orbifolds. In the case of $V$ a lattice vertex operator algebra, this becomes a special case of the more general result of [Le1], Le2], [FLM2], and [DL2], mentioned above. Permutation orbifold theory has also been studied in the physics literature, e.g., in KS and [FKS], and in [BHS], the twisted vertex operators for the generators were given for affine, Virasoro, super-Virasoro and $W_{3}$ cyclic permutation orbifolds. The characters and modular properties of permutation orbifolds are presented in [Ba]. However, the construction of twisted sectors for general permutation orbifold theory and for arbitrary orbifold theory have been open problems. The main result of this paper is the explicit construction of twisted sectors for general permutation orbifold theory. In addition, for $g$ a $k$-cycle, we show that the categories of weak, weak admissible and ordinary $g$-twisted $V^{\otimes k}$-modules are isomorphic to the categories of weak, weak admissible and ordinary $V$-modules, respectively. (The definitions of weak, weak admissible and ordinary twisted modules are given in Section 3.) Our proof includes an explicit construction of the $g$-twisted $V^{\otimes k}$-modules given a $V$-module. We show that for an arbitrary permutation automorphism $g$ of $V^{\otimes k}$ the category of weak admissible $g$-twisted modules for $V^{\otimes k}$ is semisimple and the simple objects are determined if $V$ is rational. In addition, we extend our results to the more general setting of $\gamma g$-twisted $V^{\otimes k}$-modules for $\gamma$ a general automorphism of $V$ acting diagonally on $V^{\otimes k}$ and $g$ an arbitrary permutation automorphism of $V^{\otimes k}$. One can use our constructions to calculate the characters and perform modular transformations. Moreover, we expect that the methods introduced in this paper can be extended to construct twisted sectors for arbitrary orbifold theory.

We next point out some recent results and conjectures in the theory of vertex operator algebras with which we hope to put the results of this paper into perspective and show some of the motivation which led to our results. Let $V$ be a vertex operator algebra and $g$ an automorphism of $V$. In [DM] it is shown that given a weak $V$-module $(M, Y)$, one can define a new weak $V$-module $g \circ M$ such that $g \circ M=M$ as the underlying space and the vertex operator associated to $v$ is given by $Y(g v, z)$. Then $M$ is called $g$-stable if $g \circ M$ and $M$ are isomorphic as weak $V$ modules. It is a well-known conjecture that if $V$ is rational and $g$ is of finite order, then the number of isomorphism classes of irreducible $g$-stable $V$-modules is finite and equal to the number of isomorphism classes of irreducible $g$-twisted $V$-modules. It is proved in DLM3 that if $V$ is rational and satisfies the $C_{2}$ condition, and $g$ is of finite order, then the number of isomorphism classes of irreducible $g$-twisted modules is finite and less than or equal to the number of isomorphism classes of irreducible $g$-stable $V$-modules. Moreover, if $V$ is also assumed to be $g$-rational, then the number of isomorphism classes of irreducible $g$-twisted $V$-modules is equal to the number of isomorphism classes of irreducible $g$-stable $V$-modules.

Now consider the tensor product vertex operator algebra $V^{\otimes k}$ as discussed above with $g$ a $k$-cycle. From Proposition 4.7.2 and Theorem 4.7.4 in [FHL], it follows that the number of isomorphism classes of irreducible $g$-stable $V^{\otimes k}$-modules is equal to the number of isomorphism classes of irreducible $V$-modules. Combining this result with the conjecture above, we have the conjecture that if $V$ is rational and $g$ is a $k$ cycle, then the number of isomorphism classes of irreducible $g$-twisted $V^{\otimes k}$-modules is equal to the number of isomorphism classes of irreducible $V$-modules. Thus we see that the main result in this paper is actually a stronger result than this conjecture, 
namely that the categories of weak, weak admissible and ordinary $g$-twisted $V^{\otimes k}$ modules are in fact isomorphic to the categories of weak, weak admissible and ordinary $V$-modules, respectively, even without the assumption that $V$ is rational.

To construct the isomorphism between the category of weak $g$-twisted $V^{\otimes k}$ modules and the category of weak $V$-modules for $g$ a $k$-cycle, we define a weak $g$-twisted $V^{\otimes k}$-module structure on any weak $V$-module. Our construction has been motivated by the following two results.

The first motivating result is the modular invariance of the trace function in orbifold theory DLM3]. If $V$ is a holomorphic vertex operator algebra (see [DLM3]), then $V$ is the only irreducible module for itself. Thus by Proposition 4.7.2 and Theorem 4.7.4 in [FHU, $V^{\otimes k}$ is the only irreducible $g$-stable $V^{\otimes k}$-module. Following the notation and results of [DLM3], consider the symmetric group $S_{k}$ as an automorphism group of $V^{\otimes k}$ and denote by $Z(x, y, \tau)$ the $y$-trace on the unique $x$-twisted $V^{\otimes k}$-module $V^{\otimes k}(x)$ where $x, y \in S_{k}$ commute. Then the span of $Z(x, y, \tau)$ is modular invariant. Using the modular invariance result of [DLM3], one can show that the graded dimension of irreducible $g$-twisted $V^{\otimes k}$-modules is exactly the graded dimension of $V$ except that $V_{n}$ is graded by $n / k$ (plus a uniform shift) instead of $n$ for $n \in \mathbb{Z}$. (This fact has also been observed and used in [DMVV] to study elliptic genera of symmetric products and second quantized strings.) This leads one to expect a $g$-twisted $V^{\otimes k}$-module structure on $V$ with the new gradation.

The second motivating result is the construction in [Li2] of certain $g$-twisted $V$-modules for $g$ a certain automorphism of $V$. Let $h \in V_{1}$ such that the zero-mode operator $h_{0}$ for $Y(h, z)$ acts semisimply on $V$ with only finitely many eigenvalues and such that these eigenvalues are rational. Then $e^{2 \pi h_{0} i}$ is an automorphism of $V$. Li's construction defines a new action on any $V$-module and this action gives a $e^{2 \pi h_{0} i}$-twisted $V$-module structure on the original $V$-module. The main feature in the new action is an exponential operator $\Delta(z)$ built up from the component operators $h_{n}$ of $Y(h, z)$ for $n \geq 0$. This kind of operator first appeared in the construction of twisted sectors for lattice vertex operator algebras [FLM2], [FLM3].

Now let $M$ be a weak admissible $V$-module and let $g$ be the $k$-cycle $g=(12 \cdots k)$. As suggested above from our first motivating result, one expects a weak $g$-twisted $V^{\otimes k}$-module structure on $M$ with some vertex operator $Y_{g}(v, z)$ for $v \in V^{\otimes k}$. From the twisted Jacobi identity one sees that the component operators of $Y_{g}(u \otimes \mathbf{1} \otimes$ $\cdots \otimes \mathbf{1}, z)$ for $u \in V$ form a Lie algebra. In addition, the fact that $Y_{g}(g v, z)=$ $\lim _{z^{1 / k} \rightarrow \eta^{-1} z^{1 / k}} Y_{g}(v, z)$, for $\eta=e^{-2 \pi i / k}$, indicates that all the vertex operators $Y_{g}(v, z)$ for $v \in V^{\otimes k}$ are generated by $Y_{g}(u \otimes \mathbf{1} \otimes \cdots \otimes \mathbf{1}, z)$ for $u \in V$. Therefore, the key point is to define $Y_{g}(u \otimes \mathbf{1} \otimes \cdots \otimes \mathbf{1}, z)$ which is expected to be $Y\left(\Delta_{k}(z) u, z^{1 / k}\right)$ for some operator $\Delta_{k}(z) \in($ End $V)\left[\left[z^{1 / k}, z^{-1 / k}\right]\right]$ due to our second motivating result above. In [FLM2, FLM3 and Li2] an operator $\Delta(z)$ was introduced by using vertex operators associated to certain Heisenberg algebras. But for an arbitrary vertex operator algebra, we do not have a Heisenberg algebra available. So we must find another way to construct $\Delta(z)$. Note that such a construction should also work if $V$ is the vertex operator algebra associated to the highest weight modules for the Virasoro algebra. Therefore in general, the only available operators with which we can construct $\Delta(z)$ are $L(n)$ for $n \in \mathbb{Z}$. And in fact, our operator $\Delta_{k}(z)$ is built up from $L(n)$ for $n \geq 0$ (see Section 2).

The paper is organized as follows. In Section 2, we define the operator $\Delta_{k}(z)$ on $V$ and prove several important properties of $\Delta_{k}(z)$ which are needed in subsequent 
sections. The main ideas for the proofs of these identities come from [H. In Section 3, we define a weak $g$-twisted $V^{\otimes k}$-module structure on any weak $V$-module $M$ for $g=(12 \cdots k)$ by using the operator $\Delta_{k}(z)$. As a result we construct a functor $T_{g}^{k}$ from the category of weak $V$-modules to the category of weak $g$-twisted $V^{\otimes k}$-modules such that $T_{g}^{k}$ maps weak admissible (resp., ordinary) $V$-modules into weak admissible (resp., ordinary) $g$-twisted $V^{\otimes k}$-modules. In addition, $T_{g}^{k}$ preserves irreducible objects. In Section 4, we define a weak $V$-module structure on any weak $g$-twisted $V^{\otimes k}$-module. In so doing, we construct a functor $U_{g}^{k}$ from the category of weak $g$-twisted $V^{\otimes k}$-modules to the category of weak $V$-modules such that $T_{g}^{k} \circ U_{g}^{k}=i d$ and $U_{g}^{k} \circ T_{g}^{k}=i d$. In Section 5, we give the extension of the results of Section 3 and 4 for $g$ a general $k$-cycle.

Section 6 is devoted to twisted modules for an arbitrary permutation $g \in S_{k}$. In particular we prove that if $V$ is rational, then $V^{\otimes k}$ is $g$-rational. We also construct irreducible $g$-twisted $V^{\otimes k}$-modules from irreducible $V$-modules.

In Section 7, we study various twisted modules for an automorphism of $V^{\otimes k}$ which is a product of a permutation and an automorphism of $V$. Here the automorphisms of $V$ act on $V^{\otimes k}$ diagonally. For $\gamma$ an automorphism of $V, g$ the $k$-cycle $g=(12 \cdots k)$, and $\gamma g$ an automorphism of $V^{\otimes k}$, we show that the category of weak $\gamma g$-twisted $V^{\otimes k}$-modules is isomorphic to the category of weak $\gamma^{k}$-twisted $V$ modules and that this isomorphism preserves admissible, ordinary and irreducible objects. Finally we construct $\gamma g$-twisted $V^{\otimes k}$-modules from $\gamma$-twisted $V$-modules for $g$ an arbitrary permutation and show that if $V$ is $\gamma$-rational, then $V^{\otimes k}$ is $\gamma g$ rational.

The authors thank James Lepowsky for pointing out some mistakes in earlier versions of this paper and for giving helpful comments on the paper's exposition. We would also like to thank Hirotaka Tamanoi for valuable discussions.

\section{The operator $\Delta_{k}(z)$}

In this section we define an operator $\Delta_{k}(z)=\Delta_{k}^{V}(z)$ on a vertex operator algebra $V$ for a fixed positive integer $k$. In Section 3 , we will use $\Delta_{k}(z)$ to construct a $g$ twisted $V^{\otimes k}$-module from a $V$-module where $g$ is a certain $k$-cycle.

Let $\mathbb{Z}_{+}$denote the positive integers. Let $x, y, z, z_{0}$, and $\alpha_{j}$ for $j \in \mathbb{Z}_{+}$be formal variables commuting with each other. Consider the polynomial

$$
\frac{1}{k}(1+x)^{k}-\frac{1}{k} \in x \mathbb{C}[x] .
$$

By Proposition 2.1.1 in $[\mathbb{H}]$, for any formal power series $\sum_{j \in \mathbb{Z}_{+}} c_{j} x^{j} \in x \mathbb{C}[[x]]$ there exist unique $a_{j} \in \mathbb{C}$ for $j \in \mathbb{Z}_{+}$such that

$$
\exp \left(-\sum_{j \in \mathbb{Z}_{+}} a_{j} x^{j+1} \frac{\partial}{\partial x}\right) \cdot x=\sum_{j \in \mathbb{Z}_{+}} c_{j} x^{j} .
$$

Thus for $k \in \mathbb{Z}_{+}$, we can define $a_{j} \in \mathbb{C}$ for $j \in \mathbb{Z}_{+}$, by

$$
\exp \left(-\sum_{j \in \mathbb{Z}_{+}} a_{j} x^{j+1} \frac{\partial}{\partial x}\right) \cdot x=\frac{1}{k}(1+x)^{k}-\frac{1}{k} .
$$

For example, $a_{1}=(1-k) / 2$ and $a_{2}=\left(k^{2}-1\right) / 12$. 
Let $R$ be a ring, and let $O$ be an invertible linear operator on $R\left[x, x^{-1}\right]$. We define another linear operator $O^{x \frac{\partial}{\partial x}}$ by

$$
O^{x} \frac{\partial}{\partial x} \cdot x^{n}=O^{n} x^{n}
$$

for any $n \in \mathbb{Z}$. For example, since $z^{1 / k}$ can be thought of as an invertible linear multiplication operator $\mathbb{C}\left[x, x^{-1}\right]$, we have the operator $z^{(1 / k) x \frac{\partial}{\partial x}}$ from $\mathbb{C}\left[x, x^{-1}\right]$ to $\mathbb{C}\left[z^{1 / k}, z^{-1 / k}\right]\left[x, x^{-1}\right]$. Note that $z^{(1 / k) x} \frac{\partial}{\partial x}$ can be extended to a linear operator on $\mathbb{C}\left[\left[x, x^{-1}\right]\right]$ in the obvious way.

Let

$$
\begin{aligned}
f(x) & =z^{1 / k} \exp \left(-\sum_{j \in \mathbb{Z}_{+}} a_{j} x^{j+1} \frac{\partial}{\partial x}\right) \cdot x \\
& =\exp \left(-\sum_{j \in \mathbb{Z}_{+}} a_{j} x^{j+1} \frac{\partial}{\partial x}\right) \cdot z^{(1 / k) x \frac{\partial}{\partial x}} \cdot x \\
& =\frac{z^{1 / k}}{k}(1+x)^{k}-\frac{z^{1 / k}}{k} \in z^{1 / k} x \mathbb{C}[x] .
\end{aligned}
$$

Then the compositional inverse of $f(x)$ in $x \mathbb{C}\left[z^{-1 / k}, z^{1 / k}\right][[x]]$ is given by

$$
\begin{aligned}
f^{-1}(x) & =z^{-(1 / k) x \frac{\partial}{\partial x}} \exp \left(\sum_{j \in \mathbb{Z}_{+}} a_{j} x^{j+1} \frac{\partial}{\partial x}\right) \cdot x \\
& =z^{-1 / k} \exp \left(\sum_{j \in \mathbb{Z}_{+}} a_{j} z^{-j / k} x^{j+1} \frac{\partial}{\partial x}\right) \cdot x \\
& =\left(1+k z^{-1 / k} x\right)^{1 / k}-1
\end{aligned}
$$

where the last line is considered as a formal power series in $z^{-1 / k} x \mathbb{C}\left[z^{-1 / k}\right][[x]]$, i.e., we are expanding about $x=0$ taking $1^{1 / k}=1$.

Let $f_{\alpha}(x)$ denote the formal power series

$$
f_{\alpha}(x)=z^{1 / k} \exp \left(-\sum_{j \in \mathbb{Z}_{+}} \alpha_{j} x^{j+1} \frac{\partial}{\partial x}\right) \cdot x \in z^{1 / k} x+z^{1 / k} x^{2} \mathbb{C}\left[\alpha_{1}, \alpha_{2}, \ldots\right][[x]] .
$$

Then

$$
f_{\alpha}^{-1}(x) \in z^{-1 / k} x+x^{2} \mathbb{C}[x]\left[z^{-1 / k}\right]\left[\left[\alpha_{1}, \alpha_{2}, \ldots\right]\right],
$$

and

$$
f_{\alpha}\left(f_{\alpha}^{-1}(x)+z^{-1 / k} y\right)-x \in \mathbb{C}[x]\left[z^{1 / k}, z^{-1 / k}\right]\left[\left[\alpha_{1}, \alpha_{2}, \ldots\right]\right][[y]] .
$$

Furthermore, the coefficient of the monomial $y$ in $f_{\alpha}\left(f_{\alpha}^{-1}(x)+z^{-1 / k} y\right)-x$ is in $1+x^{2} \mathbb{C}[x]\left[z^{1 / k}, z^{-1 / k}\right]\left[\left[\alpha_{1}, \alpha_{2}, \ldots\right]\right]$. Therefore, following [远], we can define

$$
\Theta_{j}=\Theta_{j}\left(\left\{-\alpha_{n}\right\}_{n \in \mathbb{Z}_{+}}, z^{1 / k}, x\right) \in \mathbb{C}[x]\left[z^{1 / k}, z^{-1 / k}\right]\left[\left[\alpha_{1}, \alpha_{2}, \ldots\right]\right]
$$

for $j \in \mathbb{N}$ by

$$
e^{\Theta_{0}} \exp \left(\sum_{j \in \mathbb{Z}_{+}} \Theta_{j} y^{j+1} \frac{\partial}{\partial y}\right) y=f_{\alpha}\left(f_{\alpha}^{-1}(x)+z^{-1 / k} y\right)-x .
$$


Proposition 2.1. $\Theta_{j}\left(\left\{-a_{n}\right\}_{n \in \mathbb{Z}_{+}}, z^{1 / k}, \frac{1}{k} z^{1 / k-1} z_{0}\right)$ for $j \in \mathbb{N}$ is well defined in $\mathbb{C}\left[z_{0}\right]\left[\left[z^{-1 / k}\right]\right]$. Furthermore

$$
\Theta_{j}\left(\left\{-a_{n}\right\}_{n \in \mathbb{Z}_{+}}, z^{1 / k}, \frac{1}{k} z^{1 / k-1} z_{0}\right)=-a_{j}\left(z+z_{0}\right)^{-j / k}
$$

for $j \in \mathbb{Z}_{+}$, and

$$
\exp \left(\Theta_{0}\left(\left\{-a_{n}\right\}_{n \in \mathbb{Z}_{+}}, z^{1 / k}, \frac{1}{k} z^{1 / k-1} z_{0}\right)\right)=z^{1 / k-1}\left(z+z_{0}\right)^{-1 / k+1},
$$

where $\left(z+z_{0}\right)^{-j / k}$ is understood to be expanded in nonnegative integral powers of $z_{0}$.

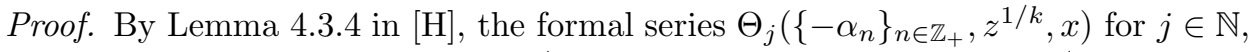
are actually in $\mathbb{C}[x]\left[\alpha_{1}, \alpha_{2}, \ldots\right]\left[\left[z^{-1 / k}\right]\right]$. Therefore $\Theta_{j}\left(\left\{-a_{n}\right\}_{n \in \mathbb{Z}_{+}}, z^{1 / k}, x\right)$ is well defined in $\mathbb{C}[x]\left[\left[z^{-1 / k}\right]\right]$, for $j \in \mathbb{N}$, and the first statement of the proposition follows.

In $y \mathbb{C}\left[z_{0}\right]\left[\left[z^{-1 / k}\right]\right][[y]]$, we have

$$
\begin{aligned}
& z^{1 / k-1}\left(z+z_{0}\right)^{-1 / k+1} \exp \left(-\sum_{j \in \mathbb{Z}_{+}} a_{j}\left(z+z_{0}\right)^{-j / k} y^{j+1} \frac{\partial}{\partial y}\right) \cdot y= \\
& =z^{1 / k-1}\left(z+z_{0}\right)^{-1 / k+1}\left(z+z_{0}\right)^{-(1 / k) y \frac{\partial}{\partial y}}\left(z+z_{0}\right)^{1 / k} \exp \left(-\sum_{j \in \mathbb{Z}_{+}} a_{j} y^{j+1} \frac{\partial}{\partial y}\right) \cdot y \\
& =z^{-1}\left(z+z_{0}\right)\left(z+z_{0}\right)^{-(1 / k) y \frac{\partial}{\partial y}} f(y) \\
& =z^{-1}\left(z+z_{0}\right) f\left(\left(z+z_{0}\right)^{-1 / k} y\right) \\
& =z^{-1}\left(z+z_{0}\right)\left(\frac{z^{1 / k}}{k}\left(1+\left(z+z_{0}\right)^{-1 / k} y\right)^{k}-\frac{z^{1 / k}}{k}\right) \\
& =\frac{z^{1 / k}}{k}\left(z^{-1 / k} y+z^{-1 / k}\left(z+k z^{1-1 / k} x\right)^{1 / k}\right)^{k}-\frac{z^{1 / k}}{k}-\left.x\right|_{x=\frac{1}{k} z^{1 / k-1} z_{0}} \\
& =f\left(z^{-1 / k}\left(z+k z^{1-1 / k} x\right)^{1 / k}-1+z^{-1 / k} y\right)-\left.x\right|_{x=\frac{1}{k} z^{1 / k-1} z_{0}} \\
& \left.\left.=f\left(f^{-1}(x)+z^{-1 / k} y\right)-\left.x\right|_{x=\frac{1}{k} z^{1 / k-1} z_{0}} y^{1 / k}, x\right)\right) \cdot \\
& =\exp \left(\Theta _ { 0 } \left(\left\{-a_{n}\right\}_{n \in \mathbb{Z}_{+}}, z^{1 / k},\right.\right. \\
& \left.\quad \exp \left(\sum_{j \in \mathbb{Z}_{+}} \Theta_{j}\left(\left\{-a_{n}\right\}_{n \in \mathbb{Z}_{+}}, z^{1 / k}, x\right) y^{j+1} \frac{\partial}{\partial y}\right) \cdot y\right|_{x=\frac{1}{k} z^{1 / k-1} z_{0}}
\end{aligned}
$$

Equations (2.1) and (2.2) follow.

Let $V=(V, Y, \mathbf{1}, \omega)$ be a vertex operator algebra. In (End $V)\left[\left[z^{1 / k}, z^{-1 / k}\right]\right]$, define

$$
\Delta_{k}^{V}(z)=\exp \left(\sum_{j \in \mathbb{Z}_{+}} a_{j} z^{-j / k} L(j)\right) k^{-L(0)} z^{(1 / k-1) L(0)} .
$$

Proposition 2.2. In (End $V$ ) $\left[\left[z^{1 / k}, z^{-1 / k}\right]\right]$, we have

$$
\Delta_{k}^{V}(z) Y\left(u, z_{0}\right) \Delta_{k}^{V}(z)^{-1}=Y\left(\Delta_{k}^{V}\left(z+z_{0}\right) u,\left(z+z_{0}\right)^{1 / k}-z^{1 / k}\right),
$$


for all $u \in V$.

Proof. By equation (5.4.10)] in [H] and Proposition 2.1 above, we have

$$
\begin{aligned}
& \Delta_{k}^{V}(z) Y\left(u, z_{0}\right) \Delta_{k}^{V}(z)^{-1}= \\
& =\exp \left(\sum_{j \in \mathbb{Z}_{+}} a_{j} z^{-j / k} L(j)\right) Y\left(k^{-L(0)} z^{(1 / k-1) L(0)} u, \frac{1}{k} z^{1 / k-1} z_{0}\right) \cdot \\
& \cdot \exp \left(-\sum_{j \in \mathbb{Z}_{+}} a_{j} z^{-j / k} L(j)\right) \\
& =z^{1 / k L(0)} \exp \left(\sum_{j \in \mathbb{Z}_{+}} a_{j} L(j)\right) z^{-(1 / k) L(0)} Y\left(k^{-L(0)} z^{(1 / k-1) L(0)} u, \frac{1}{k} z^{1 / k-1} z_{0}\right) . \\
& \cdot z^{(1 / k) L(0)} \exp \left(-\sum_{j \in \mathbb{Z}_{+}} a_{j} L(j)\right) z^{-(1 / k) L(0)} \\
& =z^{(1 / k) L(0)} Y\left(z^{-(1 / k) L(0)} \exp \left(-\sum_{j \in \mathbb{Z}_{+}} \Theta_{j}\left(\left\{-a_{n}\right\}_{n \in \mathbb{Z}_{+}}, z^{1 / k}, \frac{1}{k} z^{1 / k-1} z_{0}\right) L(j)\right)\right. \text {. } \\
& \cdot \exp \left(-\Theta_{0}\left(\left\{-a_{n}\right\}_{n \in \mathbb{Z}_{+}}, z^{1 / k}, \frac{1}{k} z^{1 / k-1} z_{0}\right) L(0)\right) \cdot k^{-L(0)} z^{(1 / k-1) L(0)} u, \\
& \left.f^{-1}\left(\frac{1}{k} z^{1 / k-1} z_{0}\right)\right) z^{-(1 / k) L(0)} \\
& =z^{(1 / k) L(0)} Y\left(z^{-(1 / k) L(0)} \exp \left(\sum_{j \in \mathbb{Z}_{+}} a_{j}\left(z+z_{0}\right)^{-j / k} L(j)\right) z^{-(1 / k-1) L(0)}\right. \text {. } \\
& \left.\cdot\left(z+z_{0}\right)^{(1 / k-1) L(0)} \cdot k^{-L(0)} z^{(1 / k-1) L(0)} u,\left(1+z^{-1} z_{0}\right)^{1 / k}-1\right) z^{-(1 / k) L(0)} \\
& =z^{(1 / k) L(0)} Y\left(z^{-(1 / k) L(0)} \Delta_{k}^{V}\left(z+z_{0}\right) u,\left(1+z^{-1} z_{0}\right)^{1 / k}-1\right) z^{-(1 / k) L(0)} \\
& =Y\left(\Delta_{k}^{V}\left(z+z_{0}\right) u,\left(z+z_{0}\right)^{1 / k}-z^{1 / k}\right)
\end{aligned}
$$

as desired.

Define $\Delta_{k}^{x}(z) \in\left(\right.$ End $\left.\mathbb{C}\left[x, x^{-1}\right]\right)\left[\left[z^{1 / k}, z^{-1 / k}\right]\right]$ by

$$
\Delta_{k}^{x}(z)=\exp \left(-\sum_{j \in \mathbb{Z}_{+}} a_{j} z^{-j / k} x^{j+1} \frac{\partial}{\partial x}\right) k^{x \frac{\partial}{\partial x}} z^{(-1 / k+1) x \frac{\partial}{\partial x}} .
$$

Proposition 2.3. In (End $\left.\mathbb{C}\left[x, x^{-1}\right]\right)\left[\left[z^{1 / k}, z^{-1 / k}\right]\right]$, we have

$$
\begin{aligned}
-\Delta_{k}^{x}(z) \frac{\partial}{\partial x}+\frac{1}{k} z^{1 / k-1} \frac{\partial}{\partial x} \Delta_{k}^{x}(z) & =\frac{\partial}{\partial z} \Delta_{k}^{x}(z) \\
-\Delta_{k}^{x}(z)^{-1} \frac{\partial}{\partial x}+k z^{-1 / k+1} \frac{\partial}{\partial x} \Delta_{k}^{x}(z)^{-1} & =k z^{-1 / k+1} \frac{\partial}{\partial z} \Delta_{k}^{x}(z)^{-1}
\end{aligned}
$$

\footnotetext{
${ }^{1}$ There is a typo in this equation in $[\mathrm{H}] . A^{(0)}$ in the first line of equation (5.4.10) should be $A^{(1)}$ which is the infinite series $\left\{A_{j}^{(1)}\right\}_{j \in \mathbb{Z}_{+}}$, where $A_{j}^{(1)} \in \mathbb{C}$. In our case, $A_{j}^{(1)}=-a_{j}$.
} 
Proof. In $\mathbb{C}\left[x, x^{-1}\right]\left[\left[z^{1 / k}, z^{-1 / k}\right]\right]$, we have

$$
\begin{aligned}
& -\Delta_{k}^{x}(z) \frac{\partial}{\partial x} \cdot x+\frac{1}{k} z^{1 / k-1} \frac{\partial}{\partial x} \Delta_{k}^{x}(z) \cdot x= \\
& =-1+\frac{1}{k} z^{1 / k-1} \frac{\partial}{\partial x}\left(k z^{-1 / k+1} z^{-(1 / k) x} \frac{\partial}{\partial x} z^{1 / k} \exp \left(-\sum_{j \in \mathbb{Z}_{+}} a_{j} x^{j+1} \frac{\partial}{\partial x}\right) \cdot x\right) \\
& =-1+\frac{1}{k} z^{1 / k-1} \frac{\partial}{\partial x}\left(k z^{-1 / k+1} z^{-(1 / k) x} \frac{\partial}{\partial x} f(x)\right) \\
& =-1+\frac{1}{k} z^{1 / k-1} k z^{-1 / k+1} \frac{\partial}{\partial x} f\left(z^{-1 / k} x\right) \\
& =-1+z^{-1 / k} f^{\prime}\left(z^{-1 / k} x\right) \\
& =-1+\left(1+z^{-1 / k} x\right)^{k-1} \\
& =-1+\left(1+z^{-1 / k} x\right)^{k}-z^{-1 / k} x\left(1+z^{-1 / k} x\right)^{k-1} \\
& =(-1+k) z^{-1 / k}\left(\frac{z^{1 / k}}{k}\left(1+z^{-1 / k} x\right)^{k}-\frac{z^{1 / k}}{k}\right) \\
& +k z^{-1 / k+1} \frac{\partial}{\partial z}\left(\frac{z^{1 / k}}{k}\left(1+z^{-1 / k} x\right)^{k}-\frac{z^{1 / k}}{k}\right) \\
& =k\left(-\frac{1}{k}+1\right) z^{-1 / k} f\left(z^{-1 / k} x\right)+k z^{-1 / k+1} \frac{\partial}{\partial z} f\left(z^{-1 / k} x\right) \\
& =\frac{\partial}{\partial z}\left(k z^{-1 / k+1} f\left(z^{-1 / k} x\right)\right) \\
& =\frac{\partial}{\partial z}\left(k z^{-1 / k+1} z^{-(1 / k) x \frac{\partial}{\partial x}} f(x)\right) \\
& =\frac{\partial}{\partial z}\left(k z^{-1 / k+1} z^{-(1 / k) x} \frac{\partial}{\partial x} z^{1 / k} \exp \left(-\sum_{j \in \mathbb{Z}_{+}} a_{j} x^{j+1} \frac{\partial}{\partial x}\right) \cdot x\right) \\
& =\frac{\partial}{\partial z}\left(k z^{-1 / k+1} \exp \left(-\sum_{j \in \mathbb{Z}_{+}} a_{j} z^{-j / k} x^{j+1} \frac{\partial}{\partial x}\right) \cdot x\right) \\
& =\frac{\partial}{\partial z} \Delta_{k}^{x}(z) \cdot x \text {. }
\end{aligned}
$$

Since $\Delta_{k}^{x}(z) \cdot x^{n}$ is well defined in $\mathbb{C}\left[x, x^{-1}\right]\left[\left[z^{1 / k}, z^{-1 / k}\right]\right]$ for all $n \in \mathbb{Z}$, by Proposition 2.1.7 in [H], we have

$$
\Delta_{k}^{x}\left(z_{2}\right) \cdot x^{n}=\left(\Delta_{k}^{x}\left(z_{2}\right) \cdot x\right)^{n}
$$


for all $n \in \mathbb{Z}$. Therefore

$$
\begin{aligned}
-\Delta_{k}^{x}(z) & \frac{\partial}{\partial x} \cdot x^{n}+\frac{1}{k} z^{1 / k-1} \frac{\partial}{\partial x} \Delta_{k}^{x}(z) \cdot x^{n}= \\
& =-n \Delta_{k}^{x}(z) \cdot x^{n-1} \frac{\partial}{\partial x} \cdot x+\frac{1}{k} z^{1 / k-1} \frac{\partial}{\partial x}\left(\Delta_{k}^{x}(z) \cdot x\right)^{n} \\
& =-n\left(\Delta_{k}^{x}(z) \cdot x^{n-1}\right)\left(\Delta_{k}^{x}(z) \frac{\partial}{\partial x} \cdot x\right) \\
& =n\left(\Delta_{k}^{x}(z) \cdot x\right)^{n-1}\left(-\Delta_{k}^{x}(z) \frac{\partial}{\partial x} \cdot x+\frac{1}{k} z^{1 / k-1}\left(\Delta_{k}^{x}(z) \cdot x\right)^{n-1} \frac{\partial}{\partial x}\left(\Delta_{k}^{x}(z) \cdot x\right)\right) \\
& =n\left(\Delta_{k}^{x}(z) \cdot x\right)^{n-1}\left(\frac{\partial}{\partial z}\left(\Delta_{k}^{x}(z) \cdot x\right)\right) \\
& =\frac{\partial}{\partial z}\left(\Delta_{k}^{x}(z) \cdot x\right)^{n} \\
& =\frac{\partial}{\partial z}\left(\Delta_{k}^{x}(z) \cdot x^{n}\right)
\end{aligned}
$$

for all $n \in \mathbb{Z}$. Equation (2.3) follows by linearity.

Similarly, in $\mathbb{C}\left[x, x^{-1}\right]\left[\left[z^{1 / k}, z^{-1 / k}\right]\right]$, we have

$$
\begin{aligned}
- & \Delta_{k}^{x}(z)^{-1} \frac{\partial}{\partial x} \cdot x+k z^{-1 / k+1} \frac{\partial}{\partial x} \Delta_{k}^{x}(z)^{-1} \cdot x= \\
& =-1+k z^{-1 / k+1} \frac{\partial}{\partial x}\left(z^{(1 / k-1) x \frac{\partial}{\partial x}} k^{-x \frac{\partial}{\partial x}} \exp \left(\sum_{j \in \mathbb{Z}_{+}} a_{j} z^{-j / k} x^{j+1} \frac{\partial}{\partial x}\right) \cdot x\right) \\
& =-1+k z^{-1 / k+1} \frac{\partial}{\partial x}\left(z^{(1 / k-1) x \frac{\partial}{\partial x}} k^{-x \frac{\partial}{\partial x}} z^{1 / k} f^{-1}(x)\right) \\
& =-1+k z \frac{\partial}{\partial x}\left(f^{-1}\left(z^{1 / k-1} k^{-1} x\right)\right) \\
& =-1+k z \frac{\partial}{\partial x}\left(\left(1+z^{-1} x\right)^{1 / k}-1\right) \\
& =-1+\left(1+z^{-1} x\right)^{1 / k-1} \\
& =\left(1+z^{-1} x\right)^{1 / k}-1-z^{-1} x\left(1+z^{-1} x\right)^{1 / k-1} \\
& =k z^{-1 / k+1}\left(\left(\frac{\partial}{\partial z} z^{1 / k}\right)\left(\left(1+z^{-1} x\right)^{1 / k}-1\right)+z^{1 / k} \frac{\partial}{\partial z}\left(\left(1+z^{-1} x\right)^{1 / k}-1\right)\right) \\
& =k z^{-1 / k+1} \frac{\partial}{\partial z}\left(z^{1 / k} f^{-1}\left(z^{1 / k-1} k^{-1} x\right)\right) \\
& =k z^{-1 / k+1} \frac{\partial}{\partial z}\left(z^{(1 / k-1) x \frac{\partial}{\partial x}} k^{-x \frac{\partial}{\partial x}} z^{1 / k} f^{-1}(x)\right) \\
& =k z^{-1 / k+1} \frac{\partial}{\partial z}\left(z^{(1 / k-1) x \frac{\partial}{\partial x}} k^{-x \frac{\partial}{\partial x}} \exp \left(\sum_{j \in \mathbb{Z}_{+}} a_{j} z^{-j / k} x^{j+1} \frac{\partial}{\partial x}\right) \cdot x\right) \\
& =k z^{-1 / k+1} \frac{\partial}{\partial z} \Delta_{k}^{x}(z)^{-1} \cdot x .
\end{aligned}
$$


The proof of identity (2.4) on $x^{n}$ for $n \in \mathbb{Z}$ is analogous to the proof of identity (2.3) on $x^{n}$ for $n \in \mathbb{Z}$. Identity (2.4) then follows by linearity.

Let $\mathfrak{L}$ be the Virasoro algebra with basis $L_{j}, j \in \mathbb{Z}$, and central charge $d \in \mathbb{C}$. The above identity can be thought of as an identity for the representation of the Virasoro algebra on $\mathbb{C}\left[x, x^{-1}\right]$ given by $L_{j} \mapsto-x^{j+1} \frac{\partial}{\partial x}$, for $j \in \mathbb{Z}$, with central charge equal to zero. We want to prove the corresponding identity for certain other representations of the Virasoro algebra, in particular for vertex operator algebras. We do this by following the method of proof used in Chapter 4 of $[\mathrm{H}]$. Letting $\kappa$ be another formal variable commuting with $z$ and $\mathfrak{L}$, we first prove the identity in $\mathcal{U}_{\Pi}(\mathfrak{L})\left[\left[z^{1 / k}, z^{-1 / k}\right]\right]\left[\left[\kappa, \kappa^{-1}\right]\right]$ where $\mathcal{U}_{\Pi}(\mathfrak{L})$ is a certain extension of the universal enveloping algebra for the Virasoro algebra, and then letting $\kappa=k$, the identity will follow in (End $V)\left[\left[z^{1 / k}, z^{-1 / k}\right]\right]$ where $V$ is a certain type of module for the Virasoro algebra.

We want to construct an extension of $\mathcal{U}(\mathfrak{L})$, the universal enveloping algebra for the Virasoro algebra, in which $\kappa^{-L_{0}}$ and $z^{(1 / k-1) L_{0}}$ can be defined. Let $V_{\Pi}$ be a vector space over $\mathbb{C}$ with basis $\left\{P_{j}: j \in \mathbb{Z}\right\}$. Let $\mathcal{T}\left(\mathfrak{L} \oplus V_{\Pi}\right)$ be the tensor algebra generated by the direct sum of $\mathfrak{L}$ and $V_{\Pi}$, and let $\mathcal{I}$ be the ideal of $\mathcal{T}\left(\mathfrak{L} \oplus V_{\Pi}\right)$ generated by

$$
\begin{gathered}
\left\{L_{i} \otimes L_{j}-L_{j} \otimes L_{i}-\left[L_{i}, L_{j}\right], L_{i} \otimes d-d \otimes L_{i}, P_{i} \otimes P_{j}-\delta_{i j} P_{i}\right. \\
\left.P_{i} \otimes d-d \otimes P_{i}, P_{i} \otimes L_{j}-L_{j} \otimes P_{i+j}: i, j \in \mathbb{Z}\right\} .
\end{gathered}
$$

Define $\mathcal{U}_{\Pi}(\mathfrak{L})=\mathcal{T}\left(\mathfrak{L} \oplus V_{\Pi}\right) / \mathcal{I}$. For any formal variable $z$ and for $n \in \mathbb{Z}$, we define

$$
z^{n L_{0}}=\sum_{j \in \mathbb{Z}} P_{j} z^{n j} \in \mathcal{U}_{\Pi}(\mathfrak{L})\left[\left[z, z^{-1}\right]\right]
$$

Note $\kappa^{-L_{0}}$ and $z^{(1 / k-1) L_{0}}$ are well-defined elements of $U_{\Pi}(\mathfrak{L})\left[\left[z^{1 / k}, z^{-1 / k}\right]\right]\left[\left[\kappa, \kappa^{-1}\right]\right]$. $\operatorname{In} \mathcal{U}_{\Pi}(\mathfrak{L})\left[\left[z^{1 / k}, z^{-1 / k}\right]\right]\left[\left[\kappa, \kappa^{-1}\right]\right]$, define

$$
\Delta_{k}^{\mathfrak{L}}(z)=\exp \left(\sum_{j \in \mathbb{Z}_{+}} a_{j} z^{-j / k} L_{j}\right) \kappa^{-L_{0}} z^{(1 / k-1) L_{0}}
$$

Proposition 2.4. In $\mathcal{U}_{\Pi}(\mathfrak{L})\left[\left[z^{1 / k}, z^{-1 / k}\right]\right]\left[\left[\kappa, \kappa^{-1}\right]\right]$, we have

$$
\begin{aligned}
\Delta_{k}^{\mathfrak{L}}(z) L_{-1}-\frac{1}{\kappa} z^{1 / k-1} L_{-1} \Delta_{k}^{\mathfrak{L}}(z) & =\frac{\partial}{\partial z} \Delta_{k}^{\mathfrak{L}}(z) \\
\Delta_{k}^{\mathfrak{L}}(z)^{-1} L_{-1}-\kappa z^{-1 / k+1} L_{-1} \Delta_{k}^{\mathfrak{L}}(z)^{-1} & =k z^{-1 / k+1} \frac{\partial}{\partial z} \Delta_{k}^{\mathfrak{L}}(z)^{-1} .
\end{aligned}
$$


Proof. In $\mathcal{U}_{\Pi}(\mathfrak{L})\left[\left[z^{1 / k}, z^{-1 / k}\right]\right]\left[\left[\kappa, \kappa^{-1}\right]\right]$, we have

$$
\begin{aligned}
& \Delta_{\hat{k}}^{\mathfrak{L}}(z) L_{-1}-\frac{1}{\kappa} z^{1 / k-1} L_{-1} \Delta_{k}^{\mathfrak{L}}(z)= \\
& =\frac{1}{\kappa} z^{1 / k-1}\left[e^{\sum_{j \in \mathbb{Z}_{+}} a_{j} z^{-j / k} L_{j}}, L_{-1}\right] \kappa^{-L_{0}} z^{(1 / k-1) L_{0}} \\
& =\frac{1}{\kappa} z^{1 / k-1} \sum_{n \in \mathbb{Z}_{+}} \frac{1}{n !}\left(\sum_{j_{1}, \ldots, j_{n} \in \mathbb{Z}_{+}} a_{j_{1}} \cdots a_{j_{n}} z^{-\left(j_{1}+\cdots+j_{n}\right) / k}\right. \\
& \left.\quad\left(\sum_{i=1, \ldots, n} L_{j_{1}} L_{j_{2}} \cdots L_{j_{i-1}}\left[L_{j_{i}}, L_{-1}\right] L_{j_{i+1}} \cdots L_{j_{n}}\right)\right) \kappa^{-L_{0}} z^{(1 / k-1) L_{0}}
\end{aligned}
$$

which is a well-defined element of $\mathcal{U}_{\Pi}(\mathfrak{L})\left[\left[z^{1 / k}, z^{-1 / k}\right]\right]\left[\left[\kappa, \kappa^{-1}\right]\right]$ involving only elements $L_{j}$ with $j \in \mathbb{N}$. The right-hand side of (2.5) also involves only $L_{j}$ for $j \in \mathbb{N}$. Thus comparing with the identity (2.3) for the representation $L_{j} \mapsto-x^{j+1} \frac{\partial}{\partial x}$, the identity (2.5) must hold. The proof of (2.6) is analogous.

Let $V$ be a module for the Virasoro algebra satisfying $V=\bigsqcup_{n \in \mathbb{Z}} V_{n}$. For $j \in \mathbb{Z}$, let $L(j) \in$ End $V$ and $c \in \mathbb{C}$ be the representation images of $L_{j}$ and $d$, respectively, for the Virasoro algebra. Assume that for $v \in V_{n}$, we have $L(0) v=n v$. For any formal variable $z$, define $z^{j L(0)} \in($ End $V)\left[\left[z, z^{-1}\right]\right]$ by

$$
z^{j L(0)} v=z^{j n} v
$$

for $v \in V_{n}$. Or equivalently, let $P(n): V \rightarrow V_{n}$ be the projection from $V$ to the homogeneous subspace of weight $n$ for $n \in \mathbb{Z}$. Then

$$
z^{j L(0)} v=\sum_{n \in \mathbb{Z}} z^{j n} P(n) v
$$

for $v \in V$. The elements $P(n) \in$ End $V$ can be thought of as the representation images of $P_{n}$ in the algebra $\mathcal{U}_{\Pi}(\mathfrak{L})$.

Note that for $k$ a positive integer, $k^{-L(0)}$ is a well-defined element of End $V$ and $z^{(1 / k-1) L(0)}$ is a well-defined element of (End $\left.V\right)\left[\left[z^{1 / k}, z^{-1 / k}\right]\right]$.

In (End $V)\left[\left[z^{1 / k}, z^{-1 / k}\right]\right]$, define

$$
\Delta_{k}^{V}(z)=\exp \left(\sum_{j \in \mathbb{Z}_{+}} a_{j} z^{-j / k} L(j)\right) k^{-L(0)} z^{(1 / k-1) L(0)} .
$$

From Proposition 4.1.1 in [ $[$ ] and Proposition 2.4, we obtain the following corollary.

Corollary 2.5. In (End $V$ ) $\left[\left[z^{1 / k}, z^{-1 / k}\right]\right]$, we have

$$
\begin{aligned}
\Delta_{k}^{V}(z) L(-1)-\frac{1}{k} z^{1 / k-1} L(-1) \Delta_{k}^{V}(z) & =\frac{\partial}{\partial z} \Delta_{k}^{V}(z) \\
\Delta_{k}^{V}(z)^{-1} L(-1)-k z^{-1 / k+1} L(-1) \Delta_{k}^{V}(z)^{-1} & =k z^{-1 / k+1} \frac{\partial}{\partial z} \Delta_{k}^{V}(z)^{-1} .
\end{aligned}
$$

In particular, the identities hold for $V$ being any vertex operator algebra. 


\section{The TWISTED SECTOR FOR $g=(12 \cdots k)$}

We first review the definitions of weak, weak admissible and ordinary $g$-twisted modules for a vertex operator algebra $V$ and an automorphism $g$ of $V$ of finite order $k$ (cf. DLM1- DLM3).

Let $(V, Y, \mathbf{1}, \omega)$ be a vertex operator algebra. A weak $g$-twisted $V=\left(V, Y_{M}\right)$ module is a $\mathbb{C}$-linear space $M$ equipped with a linear map $V \rightarrow(\operatorname{End} M)\left[\left[z^{1 / k}, z^{-1 / k}\right]\right]$, given by $v \mapsto Y_{M}(v, z)=\sum_{n \in \mathbb{Q}} v_{n} z^{-n-1}$, such that for $u, v \in V$ and $w \in M$ the following hold: (1) $v_{m} w=0$ if $m$ is sufficiently large; (2) $Y_{M}(\mathbf{1}, z)=1$; (3) $Y_{M}(v, z)=\sum_{n \in r / k+\mathbb{Z}} v_{n} z^{-n-1}$ for $v \in V^{r}$ where $V^{r}=\left\{v \in V \mid g v=e^{-2 \pi i r / k} v\right\}$; (4) the twisted Jacobi identity holds: for $u \in V^{r}$

$$
\begin{gathered}
z_{0}^{-1} \delta\left(\frac{z_{1}-z_{2}}{z_{0}}\right) Y_{M}\left(u, z_{1}\right) Y_{M}\left(v, z_{2}\right)-z_{0}^{-1} \delta\left(\frac{z_{2}-z_{1}}{-z_{0}}\right) Y_{M}\left(v, z_{2}\right) Y_{M}\left(u, z_{1}\right) \\
\quad=z_{2}^{-1}\left(\frac{z_{1}-z_{0}}{z_{2}}\right)^{-r / k} \delta\left(\frac{z_{1}-z_{0}}{z_{2}}\right) Y_{M}\left(Y\left(u, z_{0}\right) v, z_{2}\right) .
\end{gathered}
$$

It can be shown (cf. Lemma 2.2 of [DLM1, and DLM2]) that $Y_{M}(\omega, z)$ has component operators which satisfy the Virasoro algebra relations and $Y_{M}(L(-1) u, z)=$ $\frac{d}{d z} Y_{M}(u, z)$. If we take $g=1$, then we obtain a weak $V$-module.

A weak admissible $g$-twisted $V$-module is a weak $g$-twisted $V$-module $M$ which carries a $\frac{1}{k} \mathbb{Z}_{+}$-grading

$$
M=\oplus_{n \in \frac{1}{k} \mathbb{Z}_{+}} M(n)
$$

such that $v_{m} M(n) \subseteq M(n+$ wt $v-m-1)$ for homogeneous $v \in V$. We may assume that $M(0) \neq 0$ if $M \neq 0$. If $g=1$, we have a weak admissible $V$-module.

Remark 3.1. Above we used the term "weak admissible $g$-twisted module" whereas in most of the literature (cf. [DLM1], [Z]) the term "admissible $g$-twisted module" is used for this notion. We used the qualifier "weak" to stress that these are indeed only weak modules and in general are not ordinary modules. However, for the sake of brevity, we will now drop the qualifier "weak".

An (ordinary) $g$-twisted $V$-module is a weak $g$-twisted $V$-module $M$ graded by $\mathbb{C}$ induced by the spectrum of $L(0)$. That is, we have

$$
M=\coprod_{\lambda \in \mathbb{C}} M_{\lambda}
$$

where $M_{\lambda}=\{w \in M \mid L(0) w=\lambda w\}$. Moreover we require that $\operatorname{dim} M_{\lambda}$ is finite and $M_{n / k+\lambda}=0$ for fixed $\lambda$ and for all sufficiently small integers $n$. If $g=1$ we have an ordinary $V$-module.

The vertex operator algebra $V$ is called $g$-rational if every admissible $g$-twisted $V$-module is completely reducible, i.e., a direct sum of irreducible admissible $g$ twisted modules. It was proved in DLM2 that if $V$ is $g$-rational then: (1) every irreducible admissible $g$-twisted $V$-module is an ordinary $g$-twisted $V$-module; and (2) $V$ has only finitely many isomorphism classes of irreducible admissible $g$-twisted modules.

Now we turn our attention to tensor product vertex operator algebras. Let $V=(V, Y, \mathbf{1}, \omega)$ be a vertex operator algebra and $k$ a fixed positive integer as in Section 2. Then $V^{\otimes k}$ is also a vertex operator algebra (see [FHL]), and the permutation group $S_{k}$ acts naturally on $V^{\otimes k}$ as automorphisms. Let $g=(12 \cdots k)$. 
In this section we construct a functor $T_{g}^{k}$ from the category of weak $V$-modules to the category of weak $g$-twisted modules for $V^{\otimes k}$. We do this by first defining $g$-twisted vertex operators on a weak $V$-module $M$ for a set of generators which are mutually local (see [Li2]). These $g$-twisted vertex operators generate a local system which is a vertex algebra. We then construct a homomorphism of vertex algebras from $V^{\otimes k}$ to this local system which thus gives a weak $g$-twisted $V^{\otimes k}$ module structure on $M$.

For $v \in V$ denote by $v^{j} \in V^{\otimes k}$ the vector whose $j$-th tensor factor is $v$ and whose other tensor factors are $\mathbf{1}$. Then $g v^{j}=v^{j+1}$ for $j=1, \ldots, k$ where $k+1$ is understood to be 1 . Let $W$ be a weak $g$-twisted $V^{\otimes k}$-module, and let $\eta=e^{-2 \pi i / k}$. Then it follows immediately from the definition of twisted module that the $g$-twisted vertex operators on $W$ satisfy

$$
Y_{g}\left(v^{j+1}, z\right)=\lim _{z^{1 / k} \rightarrow \eta^{-j} z^{1 / k}} Y_{g}\left(v^{1}, z\right)
$$

Since $V^{\otimes k}$ is generated by $v^{j}$ for $v \in V$ and $j=1, \ldots, k$, the vertex operators $Y_{g}\left(v^{1}, z\right)$ for $v \in V$ determine all the vertex operators $Y_{g}(u, z)$ on $W$ for any $u \in$ $V^{\otimes k}$. This observation is very important in our construction of twisted sectors.

Let $u, v \in V$. Then by (3.1) the twisted Jacobi identity for $Y_{g}\left(u^{1}, z_{1}\right)$ and $Y_{g}\left(v^{1}, z_{2}\right)$ is

$$
\begin{gathered}
z_{0}^{-1} \delta\left(\frac{z_{1}-z_{2}}{z_{0}}\right) Y_{g}\left(u^{1}, z_{1}\right) Y_{g}\left(v^{1}, z_{2}\right)-z_{0}^{-1} \delta\left(\frac{z_{2}-z_{1}}{-z_{0}}\right) Y_{g}\left(v^{1}, z_{2}\right) Y_{g}\left(u^{1}, z_{1}\right) \\
=\frac{1}{k} z_{2}^{-1} \sum_{j=0}^{k-1} \delta\left(\eta^{j} \frac{\left(z_{1}-z_{0}\right)^{1 / k}}{z_{2}^{1 / k}}\right) Y_{g}\left(Y\left(g^{j} u^{1}, z_{0}\right) v^{1}, z_{2}\right)
\end{gathered}
$$

(cf. [Le2], [D]). Since $g^{j} u^{1}=u^{j+1}$, we see that $Y\left(g^{j} u^{1}, z_{0}\right) v^{1}$ only involves nonnegative integer powers of $z_{0}$ unless $j=0(\bmod k)$. Thus

$$
\left[Y_{g}\left(u^{1}, z_{1}\right), Y_{g}\left(v^{1}, z_{2}\right)\right]=\operatorname{Res}_{z_{0}} \frac{1}{k} z_{2}^{-1} \delta\left(\frac{\left(z_{1}-z_{0}\right)^{1 / k}}{z_{2}^{1 / k}}\right) Y_{g}\left(Y\left(u^{1}, z_{0}\right) v^{1}, z_{2}\right) .
$$

This shows that the component operators of $Y_{g}\left(u^{1}, z\right)$ for $u \in V$ on $W$ form a Lie algebra.

Now let $M=(M, Y)$ be a weak $V$-module. For $u \in V$ and $\Delta_{k}(z)=\Delta_{k}^{V}(z)$ given by (2.7), define

$$
\bar{Y}(u, z)=Y\left(\Delta_{k}(z) u, z^{1 / k}\right)
$$

When we put a weak $g$-twisted $V^{\otimes k}$-module structure on $M$ this $\bar{Y}(u, z)$ will be the twisted vertex operator acting on $M$ associated to $u^{1}$. Here we give several examples of $\bar{Y}(u, z)$.

If $u \in V_{n}$ is a highest weight vector then $\Delta_{k}(z) u=k^{-n} z^{(1 / k-1) n} u$ and

$$
\bar{Y}(u, z)=k^{-n} z^{(1 / k-1) n} Y\left(u, z^{1 / k}\right) .
$$

In particular, if $n=1$ we have

$$
\bar{Y}(u, z)=k^{-1} z^{1 / k-1} Y\left(u, z^{1 / k}\right) .
$$

This case is important in the study of symmetric orbifold theory for the vertex operator algebras associated to affine Lie algebras. Now we take $u=\omega$. Recall that 
$a_{2}=\left(k^{2}-1\right) / 12$. Thus

$$
\begin{aligned}
\Delta_{k}(z) \omega & =\frac{z^{2(1 / k-1)}}{k^{2}}\left(\omega+a_{2} \frac{c}{2} z^{-2 / k}\right) \\
& =\frac{z^{2(1 / k-1)}}{k^{2}}\left(\omega+\frac{\left(k^{2}-1\right) c}{24} z^{-2 / k}\right)
\end{aligned}
$$

where $c$ is the central charge. Therefore

$$
\bar{Y}(\omega, z)=\frac{z^{2(1 / k-1)}}{k^{2}} Y\left(\omega, z^{1 / k}\right)+\frac{\left(k^{2}-1\right) c}{24 k^{2}} z^{-2} .
$$

We next study the properties of the operators $\bar{Y}(u, z)$.

Lemma 3.2. For $u \in V$

$$
\bar{Y}(L(-1) u, z)=\frac{d}{d z} \bar{Y}(u, z)
$$

Proof. By Corollary 2.5, we have

$$
\begin{aligned}
\bar{Y}(L(-1) u, z) & =Y\left(\Delta_{k}(z) L(-1) u, z^{1 / k}\right) \\
& =Y\left(\frac{d}{d z} \Delta_{k}(z) u, z^{1 / k}\right)+\frac{1}{k} z^{1 / k-1} Y\left(L(-1) \Delta_{k}(z) u, z^{1 / k}\right) \\
& =Y\left(\frac{d}{d z} \Delta_{k}(z) u, z^{1 / k}\right)+\left.\frac{1}{k} z^{1 / k-1} \frac{d}{d x} Y\left(\Delta_{k}(z) u, x\right)\right|_{x=z^{1 / k}} \\
& =Y\left(\frac{d}{d z} \Delta_{k}(z) u, z^{1 / k}\right)+\left.\frac{d}{d x} Y\left(\Delta_{k}(z) u, x^{1 / k}\right)\right|_{x=z} \\
& =\frac{d}{d z} Y\left(\Delta_{k}(z) u, z^{1 / k}\right) \\
& =\frac{d}{d z} \bar{Y}(u, z)
\end{aligned}
$$

as desired.

In the proof of the following lemma and again later on, we will need some properties of the $\delta$-function. We first note that from Proposition 8.8.22 of [FLM3], for $p \in \mathbb{Z}$ we have

$$
z_{2}^{-1}\left(\frac{z_{1}-z_{0}}{z_{2}}\right)^{-p / k} \delta\left(\frac{z_{1}-z_{0}}{z_{2}}\right)=z_{1}^{-1}\left(\frac{z_{2}+z_{0}}{z_{1}}\right)^{p / k} \delta\left(\frac{z_{2}+z_{0}}{z_{1}}\right),
$$

and it is easy to see that

$$
\sum_{p=0}^{k-1}\left(\frac{z_{1}-z_{0}}{z_{2}}\right)^{p / k} z_{2}^{-1} \delta\left(\frac{z_{1}-z_{0}}{z_{2}}\right)=z_{2}^{-1} \delta\left(\frac{\left(z_{1}-z_{0}\right)^{1 / k}}{z_{2}^{1 / k}}\right) .
$$

Therefore, we have the $\delta$-function identity

$$
z_{2}^{-1} \delta\left(\frac{\left(z_{1}-z_{0}\right)^{1 / k}}{z_{2}^{1 / k}}\right)=z_{1}^{-1} \delta\left(\frac{\left(z_{2}+z_{0}\right)^{1 / k}}{z_{1}^{1 / k}}\right) .
$$

Lemma 3.3. For $u, v \in V$

$$
\left[\bar{Y}\left(u, z_{1}\right), \bar{Y}\left(v, z_{2}\right)\right]=\operatorname{Res}_{z_{0}} \frac{1}{k} z_{2}^{-1} \delta\left(\frac{\left(z_{1}-z_{0}\right)^{1 / k}}{z_{2}^{1 / k}}\right) \bar{Y}\left(Y\left(u, z_{0}\right) v, z_{2}\right) .
$$


Proof. Replacing $Y\left(u, z_{1}\right)$ and $Y\left(v, z_{2}\right)$ by $Y\left(\Delta_{k}\left(z_{1}\right) u, z_{1}^{1 / k}\right)$ and $Y\left(\Delta_{k}\left(z_{2}\right) v, z_{2}^{1 / k}\right)$, respectively, in the commutator formula

$$
\left[Y\left(u, z_{1}\right), Y\left(v, z_{2}\right)\right]=\operatorname{Res}_{x} z_{2}^{-1} \delta\left(\frac{z_{1}-x}{z_{2}}\right) Y\left(Y(u, x) v, z_{2}\right)
$$

which is a consequence of the Jacobi identity on $M$, we have

$$
\left[\bar{Y}\left(u, z_{1}\right), \bar{Y}\left(v, z_{2}\right)\right]=\operatorname{Res}_{x} z_{2}^{-1 / k} \delta\left(\frac{z_{1}^{1 / k}-x}{z_{2}^{1 / k}}\right) Y\left(Y\left(\Delta_{k}\left(z_{1}\right) u, x\right) \Delta_{k}\left(z_{2}\right) v, z_{2}^{1 / k}\right) .
$$

We want to make the change of variable $x=z_{1}^{1 / k}-\left(z_{1}-z_{0}\right)^{1 / k}$ where by $z_{1}^{1 / k}-\left(z_{1}-z_{0}\right)^{1 / k}$ we mean the power series expansion in positive powers of $z_{0}$. In this case, we note that for $n \in \mathbb{Z}$

$$
\begin{aligned}
\left.\left(z_{1}^{1 / k}-x\right)^{n}\right|_{x=z_{1}^{1 / k}-\left(z_{1}-z_{0}\right)^{1 / k}}= & \\
& =\sum_{m \in \mathbb{N}}\left(\begin{array}{c}
n \\
m
\end{array}\right)(-1)^{m} z_{1}^{n / k-m / k}\left(-\sum_{l \in \mathbb{Z}_{+}}\left(\begin{array}{c}
1 / k \\
l
\end{array}\right) z_{1}^{1 / k-l}(-1)^{l} z_{0}^{l}\right)^{m} \\
& =\sum_{m \in \mathbb{N}}\left(\begin{array}{c}
n \\
m
\end{array}\right)(-1)^{m} z_{1}^{n / k}\left(-\sum_{l \in \mathbb{Z}_{+}}\left(\begin{array}{c}
1 / k \\
l
\end{array}\right)\left(\frac{-z_{0}}{z_{1}}\right)^{l}\right)^{m} \\
& =z_{1}^{n / k}\left(1+\sum_{l \in \mathbb{Z}_{+}}\left(\begin{array}{c}
1 / k \\
l
\end{array}\right)\left(\frac{-z_{0}}{z_{1}}\right)^{l}\right)^{n} \\
& =z_{1}^{n / k}\left(1-\frac{z_{0}}{z_{1}}\right)^{n / k} \\
& =\left(z_{1}-z_{0}\right)^{n / k}
\end{aligned}
$$

Thus substituting $x=z_{1}^{1 / k}-\left(z_{1}-z_{0}\right)^{1 / k}$ into

$$
z_{2}^{-1 / k} \delta\left(\frac{z_{1}^{1 / k}-x}{z_{2}^{1 / k}}\right) Y\left(Y\left(\Delta_{k}\left(z_{1}\right) u, x\right) \Delta_{k}\left(z_{2}\right) v, z_{2}^{1 / k}\right)
$$

we have a well-defined power series given by

$$
\delta\left(\frac{\left(z_{1}-z_{0}\right)^{1 / k}}{z_{2}^{1 / k}}\right) Y\left(Y\left(\Delta_{k}\left(z_{1}\right) u, z_{1}^{1 / k}-\left(z_{1}-z_{0}\right)^{1 / k}\right) \Delta_{k}\left(z_{2}\right) v, z_{2}^{1 / k}\right) .
$$

Let $f\left(z_{1}, z_{2}, x\right)$ be a complex analytic function in $z_{1}, z_{2}$, and $x$, and let $h\left(z_{1}, z_{2}, z_{0}\right)$ be a complex analytic function in $z_{1}, z_{2}$, and $z_{0}$. Then if $f\left(z_{1}, z_{2}, h\left(z_{1}, z_{2}, z_{0}\right)\right)$ is well defined, and thinking of $z_{1}$ and $z_{2}$ as fixed, i.e., considering $f\left(z_{1}, z_{2}, h\left(z_{1}, z_{2}, z_{0}\right)\right)$ as a Laurent series in $z_{0}$, by the residue theorem of complex analysis, we have

$$
\operatorname{Res}_{x} f\left(z_{1}, z_{2}, x\right)=\operatorname{Res}_{z_{0}}\left(\frac{\partial}{\partial z_{0}} h\left(z_{1}, z_{2}, z_{0}\right)\right) f\left(z_{1}, z_{2}, h\left(z_{1}, z_{2}, z_{0}\right)\right)
$$

which of course remains true for $f$ and $h$ formal power series in their respective variables. Thus making the change of variable $x=h\left(z_{1}, z_{2}, z_{0}\right)=z_{1}^{1 / k}-\left(z_{1}-z_{0}\right)^{1 / k}$, 
using (3.11), (3.12), the $\delta$-function identity (3.9) and Proposition 2.2, we obtain

$$
\begin{aligned}
& {\left[\bar{Y}\left(u, z_{1}\right), \bar{Y}\left(v, z_{2}\right)\right]=} \\
& =\operatorname{Res}_{z_{0}} \frac{1}{k} z_{2}^{-1 / k}\left(z_{1}-z_{0}\right)^{1 / k-1} \delta\left(\frac{\left(z_{1}-z_{0}\right)^{1 / k}}{z_{2}^{1 / k}}\right) \\
& \left.=\operatorname{Res}_{z_{0}} \frac{1}{k} z_{2}^{-1} \delta\left(\frac{\left(z_{1}-z_{0}\right)^{1 / k}}{z_{2}^{1 / k}}\right) Y\left(Y\left(\Delta_{k}\left(z_{1}\right) u, z_{1}^{1 / k}-\left(z_{1}-z_{0}\right)^{1 / k}\right) z_{k}\left(z_{2}\right) v, z_{2}^{1 / k}-\left(z_{1}-z_{0}\right)^{1 / k}\right) \Delta_{k}\left(z_{2}\right) v, z_{2}^{1 / k}\right) \\
& =\operatorname{Res}_{z_{0}} \frac{1}{k} z_{1}^{-1} \delta\left(\frac{\left(z_{2}+z_{0}\right)^{1 / k}}{z_{1}^{1 / k}}\right) Y\left(Y\left(\Delta_{k}\left(z_{1}\right) u, z_{1}^{1 / k}-\left(z_{1}-z_{0}\right)^{1 / k}\right) \Delta_{k}\left(z_{2}\right) v, z_{2}^{1 / k}\right) \\
& =\operatorname{Res}_{z_{0}} \frac{1}{k} z_{2}^{-1} \delta\left(\frac{\left(z_{1}-z_{0}\right)^{1 / k}}{z_{2}^{1 / k}}\right) \\
& =\operatorname{Res}_{z_{0}} \frac{1}{k} z_{2}^{-1} \delta\left(\frac{\left(z_{1}-z_{0}\right)^{1 / k}}{z_{2}^{1 / k}}\right) Y\left(\Delta_{k}\left(z_{2}\right) Y\left(u, z_{0}\right) v, z_{2}\right) \\
& =\operatorname{Res}_{z_{0}} \frac{1}{k} z_{2}^{-1} \delta\left(\frac{\left(z_{1}-z_{0}\right)^{1 / k}}{z_{2}^{1 / k}}\right) \bar{Y}\left(Y\left(u, z_{0}\right) v, z_{2}\right),
\end{aligned}
$$

as desired.

We are now in a position to put a weak $g$-twisted $V^{\otimes k}$-module structure on $M$. For $u \in V$ set

$$
Y_{g}\left(u^{1}, z\right)=\bar{Y}(u, z) \quad \text { and } \quad Y_{g}\left(u^{j+1}, z\right)=\lim _{z^{1 / k} \rightarrow \eta^{-j} z^{1 / k}} Y_{g}\left(u^{1}, z\right) .
$$

Note that $Y_{g}\left(u^{j}, z\right)=\sum_{p=0}^{k-1} Y_{g}^{p}\left(u^{j}, z\right)$ where $Y_{g}^{p}\left(u^{j}, z\right)=\sum_{n \in p / k+\mathbb{Z}} u_{n}^{j} z^{-n-1}$.

Lemma 3.4. Let $u, v \in V$. Then

$$
\left[Y_{g}\left(u^{i}, z_{1}\right), Y_{g}\left(v^{j}, z_{2}\right)\right]=\operatorname{Res}_{z_{0}} \frac{1}{k} z_{2}^{-1} \delta\left(\frac{\eta^{j-i}\left(z_{1}-z_{0}\right)^{1 / k}}{z_{2}^{1 / k}}\right) Y_{g}\left(\left(Y\left(u, z_{0}\right) v\right)^{j}, z_{2}\right)
$$

where $\left(Y\left(u, z_{0}\right) v\right)^{j}=\sum_{n \in \mathbb{Z}}\left(u_{n} v\right)^{j} z_{0}^{-n-1}$, and

$$
\begin{aligned}
& {\left[Y_{g}^{p}\left(u^{i}, z_{1}\right), Y_{g}\left(v^{j}, z_{2}\right)\right]} \\
& \quad=\operatorname{Res}_{z_{0}} \frac{1}{k} z_{2}^{-1} \eta^{(j-i) p}\left(\frac{z_{1}-z_{0}}{z_{2}}\right)^{-p / k} \delta\left(\frac{z_{1}-z_{0}}{z_{2}}\right) Y_{g}\left(\left(Y\left(u, z_{0}\right) v\right)^{j}, z_{2}\right) .
\end{aligned}
$$

Proof. By Lemma 3.3, equation (3.14) holds if $i=j=1$. Replacing $z_{1}^{1 / k}$ and $z_{2}^{1 / k}$ by $\eta^{-i+1} z_{1}^{1 / k}$ and $\eta^{-j+1} z_{2}^{1 / k}$, respectively, we obtain equation (3.14) for any $i, j=1, \ldots, k$. Equation (3.15) is a direct consequence of (3.14).

By Lemma 3.4 for $u, v \in V$, there exists a positive integer $N$ such that

$$
\left[Y_{g}\left(u^{i}, z_{1}\right), Y_{g}\left(v^{j}, z_{2}\right)\right]\left(z_{1}-z_{2}\right)^{N}=0 .
$$


Letting $z^{1 / k}$ go to $\eta^{-i+1} z^{1 / k}$ in Lemma 3.2 , we have

$$
Y_{g}\left(L(-1) u^{i}, z\right)=\frac{d}{d z} Y_{g}\left(u^{i}, z\right)
$$

Thus the operators $Y_{g}\left(u^{i}, z\right)$ for $u \in V$, and $i=1, \ldots, k$ are mutually local and generate a local system $A$ in the sense of [Li2]. Let $\sigma$ be a map from $A$ to $A$ such that $\sigma Y_{g}\left(u^{i}, z\right)=Y_{g}\left(u^{i+1}, z\right)$ for $u \in V$ and $i=1, \ldots, k$. By Theorem 3.14 of [Li2] the local system $A$ generates a vertex algebra we denote by $\left(A, Y_{A}\right)$, and $\sigma$ extends to an automorphism of $A$ of order $k$ such that $M$ is a natural weak $\sigma$-twisted $A$ module in the sense that $Y\left(\alpha(z), z_{1}\right)=\alpha\left(z_{1}\right)$ for $\alpha(z) \in A$ are $\sigma$-twisted vertex operators on $M$.

Remark 3.5. $\sigma$ is given by

$$
\sigma a(z)=\lim _{z^{1 / k} \rightarrow \eta^{-1} z^{1 / k}} a(z)
$$

for $a(z) \in A($ see $[\mathrm{Li} 2])$.

Let $A^{i}=\left\{c(z) \in A \mid \sigma c(z)=\eta^{i} c(z)\right\}$ and $a(z) \in A^{i}$. For any integer $n$ and $b(z) \in A$, the operator $a(z)_{n} b(z)$ is an element of $A$ given by

$$
a(z)_{n} b(z)=\operatorname{Res}_{z_{1}} \operatorname{Res}_{z_{0}}\left(\frac{z_{1}-z_{0}}{z}\right)^{i / k} z_{0}^{n} \cdot X
$$

where

$$
X=z_{0}^{-1} \delta\left(\frac{z_{1}-z}{z_{0}}\right) a\left(z_{1}\right) b(z)-z_{0}^{-1} \delta\left(\frac{z-z_{1}}{-z_{0}}\right) b(z) a\left(z_{1}\right) .
$$

Or, equivalently, $a(z)_{n} b(z)$ is defined by:

$$
\sum_{n \in \mathbb{Z}}\left(a(z)_{n} b(z)\right) z_{0}^{-n-1}=\operatorname{Res}_{z_{1}}\left(\frac{z_{1}-z_{0}}{z}\right)^{i / k} \cdot X
$$

Thus following [Li2], for $a(z) \in A^{i}$, we define $Y_{A}(a(z), x)$ by setting $Y_{A}\left(a(z), z_{0}\right) b(z)$ equal to (3.18).

Lemma 3.6. We have

$$
\left[Y_{A}\left(Y_{g}\left(u^{i}, z\right), z_{1}\right), Y_{A}\left(Y_{g}\left(v^{j}, z\right), z_{2}\right)\right]=0 .
$$

Proof. From the vertex algebra structure of $A$, we have

$$
\begin{aligned}
& {\left[Y_{A}\left(Y_{g}\left(u^{i}, z\right), z_{1}\right), Y_{A}\left(Y_{g}\left(v^{j}, z\right), z_{2}\right)\right]} \\
& \quad=\operatorname{Res}_{z_{0}} z_{2}^{-1} \delta\left(\frac{z_{1}-z_{0}}{z_{2}}\right) Y_{A}\left(Y_{A}\left(Y_{g}\left(u^{i}, z\right), z_{0}\right) Y_{g}\left(v^{j}, z\right), z_{2}\right) .
\end{aligned}
$$

So we need to compute $Y_{A}\left(Y_{g}\left(u^{i}, z\right), z_{0}\right) Y_{g}\left(v^{j}, z\right)$.

Note that $Y_{g}^{p}\left(u^{i}, z\right)=\sum_{n \in p / k+\mathbb{Z}} u_{n}^{i} z^{-n-1}$ is an eigenvector for $\sigma$ with eigenvalue $\eta^{p}$. Set

$$
X_{p}=z_{0}^{-1} \delta\left(\frac{x-z}{z_{0}}\right) Y_{g}^{p}\left(u^{i}, x\right) Y_{g}\left(v^{j}, z\right)-z_{0}^{-1} \delta\left(\frac{z-x}{-z_{0}}\right) Y_{g}\left(v^{j}, z\right) Y_{g}^{p}\left(u^{i}, x\right) .
$$

\footnotetext{
${ }^{2}$ There is a typo in the statement of Theorem 3.14 in [Li2]. The $V$ in the theorem should be $A$. That is, the main result of the theorem is that the local system $A$ of the theorem has the structure of a vertex superalgebra.
} 
Then by (3.18)

$$
Y_{A}\left(Y_{g}\left(u^{i}, z\right), z_{0}\right) Y_{g}\left(v^{j}, z\right)=\sum_{p=0}^{k-1} \operatorname{Res}_{x}\left(\frac{x-z_{0}}{z}\right)^{p / k} X_{p}
$$

Using Lemma 3.4 we compute

$$
\begin{aligned}
& \operatorname{Res}_{z_{0}} z_{2}^{-1} \delta\left(\frac{z_{1}-z_{0}}{z_{2}}\right) Y_{A}\left(Y_{g}\left(u^{i}, z\right), z_{0}\right) Y_{g}\left(v^{j}, z\right)= \\
& =\operatorname{Res}_{z_{0}} \operatorname{Res}_{x} \sum_{p=0}^{k-1} z_{2}^{-1} \delta\left(\frac{z_{1}-z_{0}}{z_{2}}\right)\left(\frac{x-z_{0}}{z}\right)^{p / k} X_{p} \\
& =\operatorname{Res}_{z_{0}} \operatorname{Res}_{x} \sum_{p=0}^{k-1} \sum_{n=0}^{\infty} z_{2}^{-1} \delta\left(\frac{z_{1}-z_{0}}{z_{2}}\right)\left(\frac{x-z_{0}}{z}\right)^{p / k} \\
& =\operatorname{Res}_{z_{0}} \operatorname{Res}_{x} \sum_{p=0}^{k-1} \sum_{n=0}^{\infty} z_{2}^{-1} \delta\left(\frac{z_{1}-z_{0}}{z_{2}}\right)\left(\frac{\left.\left.x-u_{0}, x\right), Y_{g}\left(v^{j}, z\right)\right](x-z)^{n} z_{0}^{-n}}{z} z_{0}^{-1}(x-z)^{n} z_{0}^{-n}\right. \\
& \quad \operatorname{Res}_{y} \frac{1}{k} z^{-1} \eta^{(j-i) p}\left(\frac{x-y}{z}\right)^{-p / k} \delta\left(\frac{x-y}{z}\right) Y_{g}\left((Y(u, y) v)^{j}, z\right) \\
& =\operatorname{Res}_{z_{0}} \operatorname{Res}_{x} \operatorname{Res}_{y} \sum_{p=0}^{k-1} \sum_{n=0}^{\infty} z_{2}^{-1} \delta\left(\frac{z_{1}-z_{0}}{z_{2}}\right)\left(\frac{x-z_{0}}{z}\right)^{p / k} \\
& z_{0}^{-1} y^{n} z_{0}^{-n} \frac{1}{k} z^{-1} \eta^{(j-i) p}\left(\frac{x-y}{z}\right)^{-p / k} \delta\left(\frac{x-y}{z}\right) Y_{g}\left((Y(u, y) v)^{j}, z\right) .
\end{aligned}
$$

Thus using the $\delta$-function identity (3.7), we have

$$
\begin{aligned}
\operatorname{Res}_{x} z^{-1}\left(\frac{x-z_{0}}{z}\right)^{p / k}\left(\frac{x-y}{z}\right)^{-p / k} \delta\left(\frac{x-y}{z}\right)= \\
=\operatorname{Res}_{x} x^{-1}\left(\frac{x-z_{0}}{z}\right)^{p / k}\left(\frac{z+y}{x}\right)^{p / k} \delta\left(\frac{z+y}{x}\right) \\
=\operatorname{Res}_{x} x^{-1}\left(1-\frac{z_{0}}{x}\right)^{p / k} z^{-p / k}(z+y)^{p / k} \delta\left(\frac{z+y}{x}\right) \\
=\operatorname{Res}_{x} x^{-1}\left(1-\frac{z_{0}}{z+y}\right)^{p / k} z^{-p / k}(z+y)^{p / k} \delta\left(\frac{z+y}{x}\right) \\
=z^{-p / k}\left(z+y-z_{0}\right)^{p / k} .
\end{aligned}
$$


Finally, we have

$$
\begin{aligned}
& \operatorname{Res}_{z_{0}} z_{2}^{-1} \delta\left(\frac{z_{1}-z_{0}}{z_{2}}\right) Y_{A}\left(Y_{g}\left(u^{i}, z\right), z_{0}\right) Y_{g}\left(v^{j}, z\right)= \\
& =\operatorname{Res}_{y} \operatorname{Res}_{z_{0}} \frac{1}{k} \sum_{p=0}^{k-1} z_{2}^{-1} \delta\left(\frac{z_{1}-z_{0}}{z_{2}}\right) \frac{1}{z_{0}-y} \\
& =\operatorname{Res}_{y} \operatorname{Res}_{z_{0}} \frac{1}{k} \sum_{p=0}^{k-1} z_{2}^{-1} \delta\left(\frac{z_{1}-\left(z_{0}+y\right)}{z_{2}}\right) \frac{1}{z_{0}} \\
& =\operatorname{Res}_{y} \frac{1}{k} \sum_{p=0}^{k-1} z_{2}^{-1} \delta\left(\frac{\left.z_{1}-y\right) p}{z_{2}}\right) z^{-p / k}\left(z-z_{0}\right)^{p / k} Y_{g}\left((Y(u, y) v)^{j}, z\right) \\
& =0,
\end{aligned}
$$

as desired.

Lemma 3.7. For $u_{1}, \ldots, u_{k} \in V$, we have

$$
\begin{aligned}
& Y_{A}\left(Y_{g}\left(u_{k}^{k}, z\right)_{-1} \cdots Y_{g}\left(u_{2}^{2}, z\right)_{-1} Y_{g}\left(u_{1}^{1}, z\right), x\right) \\
& \quad=Y_{A}\left(Y_{g}\left(u_{k}^{k}, z\right), x\right) \cdots Y_{A}\left(Y_{g}\left(u_{2}^{2}, z\right), x\right) Y_{A}\left(Y_{g}\left(u_{1}^{1}, z\right), x\right)
\end{aligned}
$$

where $Y_{g}\left(u_{i}^{i}, z\right)_{-1}$ is the component vertex operator of $Y_{A}\left(Y\left(u_{i}^{i}, z\right), x\right)$.

Proof. The lemma follows from Lemma 3.6 above and formula (13.26) of DL1.

Define the map $f: V^{\otimes k} \rightarrow A$ by

$$
\begin{aligned}
f: V^{\otimes k} & \rightarrow A \\
u_{1} \otimes \cdots \otimes u_{k}=\left(u_{k}^{k}\right)_{-1} \cdots\left(u_{2}^{2}\right)_{-1} u_{1}^{1} & \mapsto Y_{g}\left(u_{k}^{k}, z\right)_{-1} \cdots Y_{g}\left(u_{2}^{2}, z\right)_{-1} Y_{g}\left(u_{1}^{1}, z\right)
\end{aligned}
$$

for $u_{1}, \ldots, u_{k} \in V$. Then $f\left(u^{i}\right)=Y_{g}\left(u^{i}, z\right)$.

Lemma 3.8. $f$ is a homomorphism of vertex algebras.

Proof. We need to show that

$$
f Y\left(u_{1} \otimes \cdots \otimes u_{k}, x\right)=Y_{A}\left(Y_{g}\left(u_{k}^{k}, z\right)_{-1} \cdots Y_{g}\left(u_{2}^{2}, z\right)_{-1} Y_{g}\left(u_{1}^{1}, z\right), x\right) f
$$

for $u_{i} \in V$. Take $v_{i} \in V$ for $i=1, \ldots, k$. Then

$$
\begin{aligned}
f Y\left(u_{1} \otimes \cdots \otimes u_{k},\right. & x)\left(v_{1} \otimes \cdots \otimes v_{k}\right)= \\
& =f\left(Y\left(u_{1}, x\right) v_{1} \otimes \cdots Y\left(u_{k}, x\right) v_{k}\right) \\
& =Y_{g}\left(Y\left(u_{k}^{k}, x\right) v_{k}^{k}, z\right)_{-1} \cdots Y_{g}\left(Y\left(u_{2}^{2}, x\right) v_{2}^{2}, z\right)_{-1} Y_{g}\left(Y\left(u_{1}^{1}, x\right) v_{1}^{1}, z\right) .
\end{aligned}
$$

By Lemma 3.7, we have

$$
\begin{array}{r}
Y_{A}\left(Y_{g}\left(u_{k}^{k}, z\right)_{-1} \cdots Y_{g}\left(u_{2}^{2}, z\right)_{-1} Y_{g}\left(u_{1}^{1}, z\right), x\right) f\left(v^{1} \otimes \cdots \otimes v^{k}\right) \\
=Y_{A}\left(Y_{g}\left(u_{k}^{k}, z\right), x\right) \cdots Y_{A}\left(Y_{g}\left(u_{2}^{2}, z\right), x\right) Y_{A}\left(Y_{g}\left(u_{1}^{1}, z\right), x\right) Y_{g}\left(v_{k}^{k}, z\right)_{-1} \\
\cdots Y_{g}\left(v_{2}^{2}, z\right)_{-1} Y_{g}\left(v_{1}^{1}, z\right) .
\end{array}
$$


By Lemma 3.6, it is enough to show that

$$
Y_{g}\left(Y\left(u^{i}, x\right) v^{i}, z\right)=Y_{A}\left(Y_{g}\left(u^{i}, z\right), x\right) Y_{g}\left(v^{i}, z\right)
$$

for $u, v \in V$ and $i=1, \ldots, k$. In fact, in view of the relation between $Y\left(u^{1}, z\right)$ and $Y\left(u^{i}, z\right)$ for $u \in V$, we only need to prove the case $i=1$.

By Proposition 2.2.

$$
\begin{aligned}
Y_{g}\left(Y\left(u^{1}, z_{0}\right) v^{1}, z_{2}\right) & =Y\left(\Delta_{k}\left(z_{2}\right) Y\left(u, z_{0}\right) v, z_{2}^{1 / k}\right) \\
& =Y\left(Y\left(\Delta_{k}\left(z_{2}+z_{0}\right) u,\left(z_{2}+z_{0}\right)^{1 / k}-z_{2}^{1 / k}\right) \Delta_{k}\left(z_{2}\right) v, z_{2}^{1 / k}\right) .
\end{aligned}
$$

On the other hand,

$$
Y_{A}\left(Y_{g}\left(u^{1}, z_{2}\right), z_{0}\right) Y_{g}\left(v^{1}, z_{2}\right)=\sum_{p=0}^{k-1} \operatorname{Res}_{z_{1}}\left(\frac{z_{1}-z_{0}}{z_{2}}\right)^{p / k} X
$$

where

$$
X=z_{0}^{-1} \delta\left(\frac{z_{1}-z_{2}}{z_{0}}\right) Y_{g}\left(u^{1}, z_{1}\right) Y_{g}\left(v^{1}, z_{2}\right)-z_{0}^{-1} \delta\left(\frac{z_{2}-z_{1}}{-z_{0}}\right) Y_{g}\left(v^{1}, z_{2}\right) Y_{g}\left(u^{1}, z_{1}\right) .
$$

By equation (3.16), there exists a positive integer $N$ such that

$$
\left(z_{1}-z_{2}\right)^{N} Y_{g}\left(u^{1}, z_{1}\right) Y_{g}\left(v^{1}, z_{2}\right)=\left(z_{1}-z_{2}\right)^{N} Y_{g}\left(v^{1}, z_{2}\right) Y_{g}\left(u^{1}, z_{1}\right) .
$$

Thus

$$
\begin{aligned}
X= & z_{0}^{-1} \delta\left(\frac{z_{1}-z_{2}}{z_{0}}\right) Y_{g}\left(u^{1}, z_{1}\right) Y_{g}\left(v^{1}, z_{2}\right) \\
& \quad-z_{0}^{-1} \delta\left(\frac{z_{2}-z_{1}}{-z_{0}}\right) z_{0}^{-N}\left(z_{1}-z_{2}\right)^{N} Y_{g}\left(v^{1}, z_{2}\right) Y_{g}\left(u^{1}, z_{1}\right) \\
= & z_{0}^{-1} \delta\left(\frac{z_{1}-z_{2}}{z_{0}}\right) z_{0}^{-N}\left(\left(z_{1}-z_{2}\right)^{N} Y_{g}\left(u^{1}, z_{1}\right) Y_{g}\left(v^{1}, z_{2}\right)\right) \\
& \quad-z_{0}^{-1} \delta\left(\frac{z_{2}-z_{1}}{-z_{0}}\right) z_{0}^{-N}\left(\left(z_{1}-z_{2}\right)^{N} Y_{g}\left(u^{1}, z_{1}\right) Y_{g}\left(v^{1}, z_{2}\right)\right) \\
= & z_{2}^{-1} z_{0}^{-N} \delta\left(\frac{z_{1}-z_{0}}{z_{2}}\right)\left(\left(z_{1}-z_{2}\right)^{N} Y_{g}\left(u^{1}, z_{1}\right) Y_{g}\left(v^{1}, z_{2}\right)\right)
\end{aligned}
$$

where we have used the elementary $\delta$-function relation

$$
z_{0}^{-1} \delta\left(\frac{z_{1}-z_{2}}{z_{0}}\right)-z_{0}^{-1} \delta\left(\frac{z_{2}-z_{1}}{-z_{0}}\right)=z_{2}^{-1} \delta\left(\frac{z_{1}-z_{0}}{z_{2}}\right)
$$

(cf. [FLM3]).

Therefore using the $\delta$-function relation (3.8), we have

$$
\begin{aligned}
& Y_{A}\left(Y_{g}\left(u^{1}, z_{2}\right), z_{0}\right) Y_{g}\left(v^{1}, z_{2}\right) \\
& \quad=\operatorname{Res}_{z_{1}} z_{0}^{-N} z_{2}^{-1} \delta\left(\frac{\left(z_{1}-z_{0}\right)^{1 / k}}{z_{2}^{1 / k}}\right)\left(\left(z_{1}-z_{2}\right)^{N} Y_{g}\left(u^{1}, z_{1}\right) Y_{g}\left(v^{1}, z_{2}\right)\right) .
\end{aligned}
$$


Let $x$ be a new formal variable which commutes with $z_{0}, z_{1}, z_{2}$. Then

$$
\begin{aligned}
z_{2}^{-1 / k} \delta\left(\frac{z_{1}^{1 / k}-x}{z_{2}^{1 / k}}\right)\left(\left(z_{1}-z_{2}\right)^{N} Y_{g}\left(u^{1}, z_{1}\right) Y_{g}\left(v^{1}, z_{2}\right)\right)= \\
=x^{-1} \delta\left(\frac{z_{1}^{1 / k}-z_{2}^{1 / k}}{x}\right)\left(\left(z_{1}-z_{2}\right)^{N} Y_{g}\left(u^{1}, z_{1}\right) Y_{g}\left(v^{1}, z_{2}\right)\right) \\
\quad-x^{-1} \delta\left(\frac{-z_{2}^{1 / k}+z_{1}^{1 / k}}{x}\right)\left(\left(z_{1}-z_{2}\right)^{N} Y_{g}\left(u^{1}, z_{1}\right) Y_{g}\left(v^{1}, z_{2}\right)\right) \\
=\quad\left(z_{1}-z_{2}\right)^{N} x^{-1} \delta\left(\frac{z_{1}^{1 / k}-z_{2}^{1 / k}}{x}\right) Y\left(\Delta_{k}\left(z_{1}\right) u, z_{1}^{1 / k}\right) Y\left(\Delta_{k}\left(z_{2}\right) v, z_{2}^{1 / k}\right) \\
\quad-\left(z_{1}-z_{2}\right)^{N} x^{-1} \delta\left(\frac{-z_{2}^{1 / k}+z_{1}^{1 / k}}{x}\right) Y\left(\Delta_{k}\left(z_{2}\right) v, z_{2}^{1 / k}\right) Y\left(\Delta_{k}\left(z_{1}\right) u, z_{1}^{1 / k}\right) \\
=\quad\left(z_{1}-z_{2}\right)^{N} z_{2}^{-1 / k} \delta\left(\frac{z_{1}^{1 / k}-x}{z_{2}^{1 / k}}\right) Y\left(Y\left(\Delta_{k}\left(z_{1}\right) u, x\right) \Delta_{k}\left(z_{2}\right) v, z_{2}^{1 / k}\right) .
\end{aligned}
$$

Note that the first term in the above formula is well defined when $x$ is replaced by $z_{1}^{1 / k}-\left(z_{1}-z_{0}\right)^{1 / k}$, and therefore the last term is also well defined under this substitution. Thus

$$
\begin{aligned}
& z_{0}^{-N} z_{2}^{-1 / k} \delta\left(\frac{\left(z_{1}-z_{0}\right)^{1 / k}}{z_{2}^{1 / k}}\right)\left(\left(z_{1}-z_{2}\right)^{N} Y_{g}\left(u^{1}, z_{1}\right) Y_{g}\left(v^{1}, z_{2}\right)\right)= \\
& =z_{2}^{-1 / k} \delta\left(\frac{\left(z_{1}-z_{0}\right)^{1 / k}}{z_{2}^{1 / k}}\right) Y\left(Y\left(\Delta_{k}\left(z_{1}\right) u, z_{1}^{1 / k}-\left(z_{1}-z_{0}\right)^{1 / k}\right) \Delta_{k}\left(z_{2}\right) v, z_{2}^{1 / k}\right) \\
& =z_{2}^{-1 / k} \delta\left(\frac{\left(z_{1}-z_{0}\right)^{1 / k}}{z_{2}^{1 / k}}\right) Y\left(Y\left(\Delta_{k}\left(z_{2}+z_{0}\right) u,\left(z_{2}+z_{0}\right)^{1 / k}-z_{2}^{1 / k}\right) \Delta_{k}\left(z_{2}\right) v, z_{2}^{1 / k}\right) .
\end{aligned}
$$

Finally we have

$$
\begin{aligned}
& Y_{A}\left(Y_{g}\left(u^{1}, z_{2}\right), z_{0}\right) Y_{g}\left(v^{1}, z_{2}\right)= \\
& \quad=\operatorname{Res}_{z_{1}} z_{2}^{-1} \delta\left(\frac{\left(z_{1}-z_{0}\right)^{1 / k}}{z_{2}^{1 / k}}\right) Y\left(Y\left(\Delta_{k}\left(z_{2}+z_{0}\right) u,\left(z_{2}+z_{0}\right)^{1 / k}-z_{2}^{1 / k}\right)\right. \\
& \left.\quad=Y\left(Y\left(\Delta_{k}\left(z_{2}+z_{0}\right) u,\left(z_{2}+z_{0}\right)^{1 / k}-z_{2}^{1 / k}\right) \Delta_{k}\left(z_{2}\right) v, z_{2}^{1 / k}\right) \quad \Delta_{k}\left(z_{2}\right) v, z_{2}^{1 / k}\right) \\
& \quad=Y_{g}\left(Y\left(u^{1}, z_{0}\right) v^{1}, z_{2}\right)
\end{aligned}
$$

as desired.

Let $(M, Y)$ be a weak $V$-module. Define $T_{g}^{k}(M, Y)=\left(T_{g}^{k}(M), Y_{g}\right)=\left(M, Y_{g}\right)$. That is $T_{g}^{k}(M, Y)$ is $M$ as the underlying vector space and the vertex operator $Y_{g}$ is given by (3.13).

Now we state our first main theorem of the paper. 
Theorem 3.9. $\left(T_{g}^{k}(M), Y_{g}\right)$ is a weak g-twisted $V^{\otimes k}$-module such that $T_{g}^{k}(M)=$ $M$, and $Y_{g}$, defined by 3.13 , is the linear map from $V^{\otimes k}$ to

$$
\left(\operatorname{End} T_{g}^{k}(M)\right)\left[\left[z^{1 / k}, z^{-1 / k}\right]\right]
$$

defining the twisted module structure. Moreover,

(1) $(M, Y)$ is an irreducible weak $V$-module if and only if $\left(T_{g}^{k}(M), Y_{g}\right)$ is an irreducible weak $g$-twisted $V^{\otimes k}$-module.

(2) $M$ is an admissible $V$-module if and only if $T_{g}^{k}(M)$ is an admissible $g$-twisted $V^{\otimes k}$-module.

(3) $M$ is an ordinary $V$-module if and only if $T_{g}^{k}(M)$ is an ordinary $g$-twisted $V^{\otimes k}$-module.

Proof. It is immediate from Lemma 3.8 that $T_{g}^{k}(M)=M$ is a weak $g$-twisted $V^{\otimes k}$-module with $Y_{g}\left(u^{1}, z\right)=\bar{Y}(u, z)$. Note that

$$
Y_{g}\left(\left(\Delta_{k}(z)^{-1} u\right)^{1}, z\right)=\bar{Y}\left(\Delta_{k}(z)^{-1} u, z\right)=Y\left(u, z^{1 / k}\right)
$$

and that all twisted vertex operators $Y_{g}(v, z)$ for $v \in V^{\otimes k}$ can be generated from $Y_{g}\left(u^{1}, z\right)$ for $u \in V$. It is clear now that $M$ is an irreducible weak $V$-module if and only if $T_{g}^{k}(M)$ is an irreducible weak $g$-twisted $V^{\otimes k}$-module. So (1) has been proved.

For (2) since $M$ is an admissible $V$-module, we have $M=\oplus_{n \in \mathbb{N}} M(n)$ such that for $m \in \frac{1}{k} \mathbb{Z}$, the component operator $u_{m}$ satisfies $u_{m} M(n) \subset M($ wt $u-m-1+n)$ if $u \in V$ is of homogeneous weight. Define a $\frac{1}{k} \mathbb{Z}_{+}$-gradation on $T_{g}^{k}(M)$ such that $T_{g}^{k}(M)(n / k)=M(n)$ for $n \in \mathbb{Z}$. Recall that $Y_{g}(v, z)=\sum_{m \in \frac{1}{k} \mathbb{Z}} v_{n} z^{-m-1}$ for $v \in$ $V^{\otimes k}$. We have to show that $v_{m} T_{g}^{k}(M)(n) \subset T_{g}^{k}(M)($ wt $v-m-1+n)$ for $m, n \in \frac{1}{k} \mathbb{Z}$. As before, since all twisted vertex operators $Y_{g}(v, z)$ for $v \in V^{\otimes k}$ can be generated from $Y_{g}\left(u^{1}, z\right)$ for $u \in V$, it is enough to show $u_{m}^{1} T_{g}^{k}(M)(n) \subset T_{g}^{k}(M)($ wt $u-m-$ $1+n)$.

Let $u \in V_{p}$ for $p \in \mathbb{Z}$. Then

$$
\Delta_{k}(z) u=\sum_{i=0}^{\infty} u(i) z^{1 / k-p-i / k}
$$

where $u(i) \in V_{p-i}$. Thus

$$
\left.Y_{g}\left(u^{1}, z\right)=Y\left(\Delta_{k}(z) u, z^{1 / k}\right)=\sum_{i=0}^{\infty} Y\left(u(i), z^{1 / k}\right)\right) z^{1 / k-p-i / k}
$$

and for $m \in \frac{1}{k} \mathbb{Z}$

$$
u_{m}^{1}=\sum_{i=0}^{\infty} u(i)_{(1-k) p-i-1+k m+k} .
$$

Since the weight of $u(i)_{(1-k) p-i-1+k m+k}$ is $k(p-m-1)$, we see that $u_{m}^{1} T_{g}^{k}(M)(n)=$ $u_{m}^{1} M(k n) \subset M(k(p-m-1+n))$. That is $u_{m}^{1} T_{g}^{k}(M)(n) \subset T_{g}^{k}(M)(p-m-1+n)$, showing that $T_{g}^{k}(M)$ is an admissible $g$-twisted $V^{\otimes k}$-module.

Similarly, one can show that if $T_{g}^{k}(M)$ is an admissible $g$-twisted $V^{\otimes k}$-module, then $M$ is an admissible $V$-module with $M(n)=T_{g}^{k}(M)(n / k)$ for $n \in \mathbb{Z}$. 
In order to prove (3) we write $Y_{g}(\bar{\omega}, z)=\sum_{n \in \mathbb{Z}} L_{g}(n) z^{-n-2}$ where $\bar{\omega}=\sum_{j=1}^{k} \omega^{j}$. We have

$$
Y_{g}(\bar{\omega}, z)=\sum_{i=0}^{k-1} \lim _{z^{1 / k} \mapsto \eta^{-i} z^{1 / k}} Y_{g}\left(\omega^{1}, z\right) .
$$

It follows from $(3.6)$ that $L_{g}(0)=\frac{1}{k} L(0)+\frac{\left(k^{2}-1\right) c}{24 k}$. This immediately implies (3).

Let $V$ be an arbitrary vertex operator algebra and $g$ an automorphism of $V$ of finite order. We denote the categories of weak, admissible and ordinary $g$-twisted $V$-modules by $\mathcal{C}_{w}^{g}(V), \mathcal{C}_{a}^{g}(V)$ and $\mathcal{C}^{g}(V)$, respectively. If $g=1$, we habitually remove the index $g$.

Now again consider the vertex operator $V^{\otimes k}$ and the $k$-cycle $g=(12 \cdots k)$. Define

$$
\begin{aligned}
T_{g}^{k}: \mathcal{C}_{w}(V) & \longrightarrow \mathcal{C}_{w}^{g}\left(V^{\otimes k}\right) \\
(M, Y) & \mapsto\left(T_{g}^{k}(M), Y_{g}\right)=\left(M, Y_{g}\right) \\
f & \mapsto T_{g}^{k}(f)=f
\end{aligned}
$$

for $(M, Y)$ an object and $f$ a morphism in $\mathcal{C}_{w}(V)$.

The following corollary to Theorem 3.9 is obvious.

Corollary 3.10. $T_{g}^{k}$ is a functor from the category $\mathcal{C}_{w}(V)$ to the category $\mathcal{C}_{w}^{g}\left(V^{\otimes k}\right)$ such that: (1) $T_{g}^{k}$ preserves irreducible objects; (2) The restrictions of $T_{g}^{k}$ to $\mathcal{C}_{a}(V)$ and $\mathcal{C}(V)$ are functors from $\mathcal{C}_{a}(V)$ and $\mathcal{C}(V)$ to $\mathcal{C}_{a}^{g}\left(V^{\otimes k}\right)$ and $\mathcal{C}^{g}\left(V^{\otimes k}\right)$, respectively.

In the next section we will construct a functor $U_{g}^{k}$ from the category $\mathcal{C}_{w}^{g}\left(V^{\otimes k}\right)$ to the category $\mathcal{C}_{w}(V)$ such that $U_{g}^{k} \circ T_{g}^{k}=i d_{\mathcal{C}_{w}(V)}$ and $\left.T_{g}^{k} \circ U_{g}^{k}=i d_{\mathcal{C}_{w}^{g}(V \otimes k}\right)$.

Remark 3.11. In constructing the weak $g$-twisted $V^{\otimes k}$-module structure on the weak $V$-module $(M, Y)$, we chose to define $Y_{g}\left(u^{1}, z\right)$ by (3.13) which then generate $Y_{g}(v, z)$ for all $v \in V^{\otimes k}$. But our choice of $u^{1}$ for $u \in V$ as generators is not canonical. We could just as well have chosen to define $Y_{g}^{j}\left(u^{j}, z\right)=\bar{Y}(u, z)$ for $j=2, \ldots, k$, and generated $g$-twisted operators $Y_{g}^{j}(v, z)$ for $v \in V^{\otimes k}$ from these rather than from $Y_{g}^{1}\left(u^{1}, z\right)$. These new $g$-twisted operators $Y_{g}^{j}(v, z)$ are related to the old ones $Y_{g}=Y_{g}^{1}$ by

$$
Y_{g}^{j+1}(v, z)=\lim _{z^{1 / k} \rightarrow \eta^{j} z^{1 / k}} Y_{g}(v, z)
$$

for $j=1, \ldots, k-1$. This is a reflection of the fact that for any vertex operator algebra $(V, Y, \mathbf{1}, \omega)$ and any automorphism $g$ of $V$, if $\left(M, Y_{g}\right)$ is a weak $g$-twisted $V$-module, then so is $\left(M, \tilde{Y}_{g}\right)$ where

$$
\tilde{Y}_{g}(v, z)=\lim _{z^{1 / k} \rightarrow \eta^{j}} z^{1 / k} Y_{g}(v, z) .
$$

Furthermore, if $\left(M, Y_{g}\right)$ is admissible, then $\left(M, Y_{g}\right)$ and $\left(M, \tilde{Y}_{g}\right)$ are isomorphic as $g$-twisted $V$-modules via

$$
\begin{aligned}
f:\left(M, Y_{g}\right) & \rightarrow\left(M, \tilde{Y}_{g}\right) \\
w & \mapsto \eta^{j k n} w
\end{aligned}
$$


for $w \in M(n)$. (Note that here we have used the fact that $\eta^{k(\mathrm{wt} v)}=1$ for all $v \in V$ of homogeneous weight wt $v$.)

\section{Constructing a Weak $V$-module Structure on a Weak $g=(12 \cdots k)$-TWISTED $V^{\otimes k}$-MODULE}

For $k \in \mathbb{Z}_{+}$and $g=(12 \cdots k)$, let $M=\left(M, Y_{g}\right)$ be a weak $g$-twisted $V^{\otimes k_{-}}$ module. Motivated by the construction of weak $g$-twisted $V^{\otimes k}$-modules from weak $V$-modules in Section 3, we consider

$$
Y_{g}\left(\left(\Delta_{k}\left(z^{k}\right)^{-1} u\right)^{1}, z^{k}\right)
$$

for $u \in V$ where $\Delta_{k}(z)^{-1}=\Delta_{k}^{V}(z)^{-1}$ is given by (2.7), i.e.,

$$
\Delta_{k}(z)^{-1}=z^{-(1 / k-1) L(0)} k^{L(0)} \exp \left(-\sum_{j \in \mathbb{Z}_{+}} a_{j} z^{-j / k} L(j)\right) .
$$

Note that (4.1) is multivalued since $Y_{g}\left(\left(\Delta_{k}(z)^{-1} u\right)^{1}, z\right) \in(\operatorname{End} M)\left[\left[z^{1 / k}, z^{-1 / k}\right]\right]$. We thus define $Y_{U}(u, z)=Y_{g}\left(\left(\Delta_{k}\left(z^{k}\right)^{-1} u\right)^{1}, z^{k}\right)$ to be the unique formal Laurent series in $(\operatorname{End} M)\left[\left[z, z^{-1}\right]\right]$ given by taking $\left(z^{k}\right)^{1 / k}=z$. Our goal in this section is to construct a functor $U_{g}^{k}: \mathcal{C}_{w}^{g}\left(V^{\otimes k}\right) \rightarrow \mathcal{C}_{w}(V)$ with $U_{g}^{k}(M, Y)=\left(U_{g}^{k}(M), Y_{U}\right)=$ $\left(M, Y_{U}\right)$. If we instead define $Y_{U}$ by taking $\left(z^{k}\right)^{1 / k}=\eta^{j} z$ for $\eta=e^{-2 \pi i / k}$ with $j=1, \ldots, k-1$, then $\left(M, Y_{U}\right)$ will not be a weak $V$-module. Further note that this implies that if we allow $z$ to be complex number and if we define $z^{1 / k}$ using the principal branch of the logarithm, then much of our work in this section is valid if and only if $-\pi / k<\arg z<\pi / k$.

Lemma 4.1. For $u \in V$, we have

$$
\begin{aligned}
Y_{U}(L(-1) u, z) & =\left(\frac{d}{d z}\left(\left(z^{k}\right)^{1 / k}\right)\right) \frac{d}{d z} Y_{U}(u, z) \\
& =\frac{d}{d z} Y_{U}(u, z)
\end{aligned}
$$

on $U_{g}^{k}(M)=M$. Thus the $L(-1)$-derivative property holds for $Y_{U}$.

Proof. The proof is similar to that of Lemma 3.2. By Corollary 2.5 we have

$$
\Delta_{k}(z)^{-1} L(-1)-k z^{-1 / k+1} L(-1) \Delta_{k}(z)^{-1}=k z^{-1 / k+1} \frac{d}{d z} \Delta_{k}(z)^{-1} .
$$

Making the change of variable $z \rightarrow z^{k}$ gives

$$
\Delta_{k}\left(z^{k}\right)^{-1} L(-1)-k\left(z^{k}\right)^{-1 / k} z^{k} L(-1) \Delta_{k}\left(z^{k}\right)^{-1}=\left(z^{k}\right)^{-1 / k} z \frac{d}{d z} \Delta_{k}\left(z^{k}\right)^{-1} .
$$

Thus if $\left(z^{k}\right)^{1 / k}=\eta^{j} z$, we have

$$
\begin{aligned}
& \frac{d}{d z} Y_{g}\left(\left(\Delta_{k}\left(z^{k}\right)^{-1} u\right)^{1}, z^{k}\right)= \\
& \quad=Y_{g}\left(\left(\frac{d}{d z} \Delta_{k}\left(z^{k}\right)^{-1} u\right)^{1}, z^{k}\right)+\left.\frac{d}{d x} Y_{g}\left(\left(\Delta_{k}\left(z^{k}\right)^{-1} u\right)^{1}, x^{k}\right)\right|_{x=z} \\
& \quad=Y_{g}\left(\left(\frac{d}{d z} \Delta_{k}\left(z^{k}\right)^{-1} u\right)^{1}, z^{k}\right)+k z^{k-1} Y_{g}\left(L(-1)\left(\Delta_{k}\left(z^{k}\right)^{-1} u\right)^{1}, z^{k}\right) \\
& \quad=Y_{g}\left(\left(\frac{d}{d z} \Delta_{k}\left(z^{k}\right)^{-1} u\right)^{1}, z^{k}\right)+k z^{k-1} Y_{g}\left(\left(L(-1) \Delta_{k}\left(z^{k}\right)^{-1} u\right)^{1}, z^{k}\right) \\
& \quad=\eta^{j} Y_{g}\left(\left(\Delta_{k}\left(z^{k}\right)^{-1} L(-1) u\right)^{1}, z^{k}\right) .
\end{aligned}
$$


Since by definition $Y_{U}(u, z)=Y_{g}\left(\left(\Delta_{k}\left(z^{k}\right)^{-1} u\right)^{1}, z^{k}\right)$ with $\left(z^{k}\right)^{1 / k}=z$, the result follows.

Lemma 4.2. Let $u, v \in V$. Then in $U_{g}^{k}(M)=M$,

$$
\left[Y_{U}\left(u, z_{1}\right), Y_{U}\left(v, z_{2}\right)\right]=\operatorname{Res}_{z_{0}} z_{2}^{-1} \delta\left(\frac{z_{1}-z_{0}}{z_{2}}\right) Y_{U}\left(Y\left(u, z_{0}\right) v, z_{2}\right) .
$$

Proof. The proof is similar to the proof of Lemma 3.3. From the twisted Jacobi identity, we have

$$
\left[Y_{g}\left(u^{1}, z_{1}\right), Y_{g}\left(v^{1}, z_{2}\right)\right]=\operatorname{Res}_{z_{0}} \frac{1}{k} z_{2}^{-1} \delta\left(\frac{\left(z_{1}-z_{0}\right)^{1 / k}}{z_{2}^{1 / k}}\right) Y_{g}\left(Y\left(u^{1}, z_{0}\right) v^{1}, z_{2}\right) .
$$

Therefore,

$$
\begin{aligned}
& {\left[Y_{U}\left(u, z_{1}\right), Y_{U}\left(v, z_{2}\right)\right]=} \\
& \quad=\left[Y_{g}\left(\left(\Delta_{k}\left(z_{1}^{k}\right)^{-1} u\right)^{1}, z_{1}^{k}\right), Y_{g}\left(\left(\Delta_{k}\left(z_{2}^{k}\right)^{-1} v\right)^{1}, z_{2}^{k}\right)\right] \\
& \quad=\operatorname{Res}_{x} \frac{1}{k} z_{2}^{-k} \delta\left(\frac{\left(z_{1}^{k}-x\right)^{1 / k}}{z_{2}}\right) Y_{g}\left(Y\left(\left(\Delta_{k}\left(z_{1}^{k}\right)^{-1} u\right)^{1}, x\right)\left(\Delta_{k}\left(z_{2}^{k}\right)^{-1} v\right)^{1}, z_{2}^{k}\right) .
\end{aligned}
$$

We want to make the change of variable $x=z_{1}^{k}-\left(z_{1}-z_{0}\right)^{k}$ where we choose $z_{0}$ such that $\left(\left(z_{1}-z_{0}\right)^{k}\right)^{1 / k}=z_{1}-z_{0}$. Then noting that $\left.\left(z_{1}^{k}-x\right)^{n / k}\right|_{x=z_{1}^{k}-\left(z_{1}-z_{0}\right)^{k}}=$ $\left(z_{1}-z_{0}\right)^{n}$ for all $n \in \mathbb{Z}$, and using (3.12), we have

$$
\begin{aligned}
& {\left[Y_{U}\left(u, z_{1}\right), Y_{U}\left(v, z_{2}\right)\right]=} \\
& =\operatorname{Res}_{z_{0}} z_{2}^{-k}\left(z_{1}-z_{0}\right)^{k-1} \delta\left(\frac{z_{1}-z_{0}}{z_{2}}\right) Y_{g}\left(Y\left(\left(\Delta_{k}\left(z_{1}^{k}\right)^{-1} u\right)^{1}, z_{1}^{k}-\left(z_{1}-z_{0}\right)^{k}\right)\right. \\
& \left.\quad\left(\Delta_{k}\left(z_{2}^{k}\right)^{-1} v\right)^{1}, z_{2}^{k}\right) \\
& =\operatorname{Res}_{z_{0}} z_{2}^{-1} \delta\left(\frac{z_{1}-z_{0}}{z_{2}}\right) Y_{g}\left(Y\left(\left(\Delta_{k}\left(z_{1}^{k}\right)^{-1} u\right)^{1}, z_{1}^{k}-\left(z_{1}-z_{0}\right)^{k}\right)\left(\Delta_{k}\left(z_{2}^{k}\right)^{-1} v\right)^{1}, z_{2}^{k}\right) \\
& =\operatorname{Res}_{z_{0}} z_{2}^{-1} \delta\left(\frac{z_{1}-z_{0}}{z_{2}}\right) Y_{g}\left(\left(Y\left(\Delta_{k}\left(\left(z_{2}+z_{0}\right)^{k}\right)^{-1} u,\left(z_{2}+z_{0}\right)^{k}-z_{2}^{k}\right)\right.\right. \\
& \left.\left.\Delta_{k}\left(z_{2}^{k}\right)^{-1} v\right)^{1}, z_{2}^{k}\right) .
\end{aligned}
$$

Thus the proof is reduced to proving

$$
Y\left(\Delta_{k}\left(\left(z_{2}+z_{0}\right)^{k}\right)^{-1} u,\left(z_{2}+z_{0}\right)^{k}-z_{2}^{k}\right) \Delta_{k}\left(z_{2}^{k}\right)^{-1}=\Delta_{k}\left(z_{2}^{k}\right)^{-1} Y\left(u, z_{0}\right),
$$

i.e., proving

$$
\Delta_{k}\left(z_{2}^{k}\right) Y\left(\Delta_{k}\left(\left(z_{2}+z_{0}\right)^{k}\right)^{-1} u,\left(z_{2}+z_{0}\right)^{k}-z_{2}^{k}\right) \Delta_{k}\left(z_{2}^{k}\right)^{-1}=Y\left(u, z_{0}\right) .
$$

In Proposition 2.2, substituting $u, z$ and $z_{0}$ by $\Delta_{k}\left(\left(z_{2}+z_{0}\right)^{k}\right)^{-1} u, z_{2}^{k}$ and $\left(z_{2}+\right.$ $\left.z_{0}\right)^{k}-z_{2}^{k}$, respectively, gives equation (4.3).

Theorem 4.3. With the notations as above, $U_{g}^{k}\left(M, Y_{g}\right)=\left(U_{g}^{k}(M), Y_{U}\right)=\left(M, Y_{U}\right)$ is a weak $V$-module.

Proof. Since the $L(-1)$-derivation property has been proved for $Y_{U}$ in Lemma 4.1 , we only need to prove the Jacobi identity which is equivalent to the commutator formula given by Lemma 4.2 and the associator formula which states that for $u, v \in$ $V$ and $w \in U_{g}^{k}(M)$ there exists a positive integer $n$ such that

$$
\left(z_{0}+z_{2}\right)^{n} Y_{U}\left(u, z_{0}+z_{2}\right) Y_{U}\left(v, z_{2}\right) w=\left(z_{2}+z_{0}\right)^{n} Y_{U}\left(Y\left(u, z_{0}\right) v, z_{2}\right) w
$$


Write $u^{1}=\sum_{i=0}^{k-1} u_{(i)}^{1}$ where $g u_{(i)}^{1}=\eta^{i} u_{(i)}^{1}$. Then from the twisted Jacobi identity, we have the following associator: there exists a positive integer $m$ such that for $n \geq m$,

$$
\left(z_{0}+z_{2}\right)^{i / k+n} Y_{g}\left(u_{(i)}^{1}, z_{0}+z_{2}\right) Y_{g}\left(v^{1}, z_{2}\right) w=\left(z_{2}+z_{0}\right)^{i / k+n} Y_{g}\left(Y\left(u_{(i)}^{1}, z_{0}\right) v^{1}, z_{2}\right) w
$$

for $i=0, \ldots, k-1$. Replacing $z_{2}$ by $z_{2}^{k}$ and $z_{0}$ by $\left(z_{0}+z_{2}\right)^{k}-z_{2}^{k}$ gives

$$
\begin{aligned}
& \left(z_{0}+z_{2}\right)^{i+k n} Y_{g}\left(u_{(i)}^{1},\left(z_{0}+z_{2}\right)^{k}\right) Y_{g}\left(v^{1}, z_{2}^{k}\right) w \\
& \quad=\left(z_{2}+z_{0}\right)^{i+k n} Y_{g}\left(Y\left(u_{(i)}^{1},\left(z_{2}+z_{0}\right)^{k}-z_{2}^{k}\right) v^{1}, z_{2}^{k}\right) w .
\end{aligned}
$$

Note that if $a \in V^{\otimes k}$ such that $g a=\eta^{i} a$, then $Y_{g}(a, z)=\sum_{l \in i / k+\mathbb{Z}} a_{n} z^{-l-1}$. Thus there exists a positive integer $m_{i}$ such that if $n_{i} \geq m_{i}$, then

$$
\begin{aligned}
\left(z_{0}+z_{2}\right)^{n_{i}} Y_{g}\left(u_{(i)}^{1},\left(z_{0}+z_{2}\right)^{k}\right) & Y_{g}\left(v^{1}, z_{2}^{k}\right) w \\
& =\left(z_{2}+z_{0}\right)^{n_{i}} Y_{g}\left(Y\left(u_{(i)}^{1},\left(z_{2}+z_{0}\right)^{k}-z_{2}^{k}\right) v^{1}, z_{2}^{k}\right) w
\end{aligned}
$$

for $i=0, \ldots, k-1$. As a result we see that there exists a positive integer $m$ such that if $n \geq m$, then

$$
\begin{aligned}
\left(z_{0}+z_{2}\right)^{n} Y_{g}\left(u^{1},\left(z_{0}+z_{2}\right)^{k}\right) Y_{g}\left(v^{1}, z_{2}^{k}\right) w & \\
& =\left(z_{2}+z_{0}\right)^{n} Y_{g}\left(Y\left(u^{1},\left(z_{2}+z_{0}\right)^{k}-z_{2}^{k}\right) v^{1}, z_{2}^{k}\right) w .
\end{aligned}
$$

Now write $\Delta_{k}\left(\left(z_{0}+z_{2}\right)^{k}\right)^{-1} u=\sum_{j \in \mathbb{N}} u_{j}\left(z_{0}+z_{2}\right)^{s_{j}}$ for some $u_{j} \in V$ and integers $s_{j}$, and note that this is a finite sum. Similarly we have a finite sum $\Delta_{k}\left(z_{2}^{k}\right)^{-1} v=$ $\sum_{j \in \mathbb{N}} v_{j} z_{2}^{t_{j}}$ for some $v_{j} \in V$ and $t_{j} \in \mathbb{Z}$. Thus there exists a positive integer $m$ such that if $n \geq m$, then

$$
\begin{aligned}
\left(z_{0}+z_{2}\right)^{n+s_{i}} Y_{g}\left(u_{i}^{1},\left(z_{0}+z_{2}\right)^{k}\right) & Y_{g}\left(v_{j}^{1}, z_{2}^{k}\right) w \\
& =\left(z_{2}+z_{0}\right)^{n+s_{i}} Y_{g}\left(Y\left(u_{i}^{1},\left(z_{2}+z_{0}\right)^{k}-z_{2}^{k}\right) v_{j}^{1}, z_{2}^{k}\right) w
\end{aligned}
$$

for all $i, j \in \mathbb{N}$. Finally we have for $n \geq m$,

$$
\begin{aligned}
\left(z_{0}\right. & \left.+z_{2}\right)^{n} Y_{U}\left(u, z_{0}+z_{2}\right) Y_{U}\left(v, z_{2}\right) w= \\
& =\left(z_{2}+z_{0}\right)^{n} Y_{g}\left(\left(\Delta_{k}\left(\left(z_{0}+z_{2}\right)^{k}\right)^{-1} u\right)^{1},\left(z_{0}+z_{2}\right)^{k}\right) Y_{g}\left(\left(\Delta_{k}\left(z_{2}^{k}\right)^{-1} v\right)^{1}, z_{2}^{k}\right) w \\
& =\sum_{i, j \geq 0}\left(z_{0}+z_{2}\right)^{n+s_{i}} z_{2}^{t_{j}} Y_{g}\left(u_{i}^{1},\left(z_{0}+z_{2}\right)^{k}\right) Y_{g}\left(v_{j}^{1}, z_{2}^{k}\right) w \\
& =\sum_{i, j \geq 0}\left(z_{2}+z_{0}\right)^{n+s_{i}} z_{2}^{t_{j}} Y_{g}\left(Y\left(u_{i}^{1},\left(z_{2}+z_{0}\right)^{k}-z_{2}^{k}\right) v_{j}^{1}, z_{2}^{k}\right) w \\
& \left.=\left(z_{2}+z_{0}\right)^{n} Y_{g}\left(Y\left(\Delta_{k}\left(\left(z_{2}+z_{0}\right)^{k}\right)^{-1} u\right)^{1},\left(z_{2}+z_{0}\right)^{k}-z_{2}^{k}\right)\left(\Delta_{k}\left(z_{2}^{k}\right)^{-1} v\right)^{1}, z_{2}^{k}\right) w \\
& =\left(z_{2}+z_{0}\right)^{n} Y_{g}\left(\left(\Delta_{k}\left(z_{2}^{k}\right)^{-1} Y\left(u, z_{0}\right) v\right)^{1}, z_{2}^{k}\right) w \\
& =\left(z_{2}+z_{0}\right)^{n} Y_{U}\left(Y\left(u, z_{0}\right) v, z_{2}\right) w
\end{aligned}
$$

where we have used equation (4.3), completing the proof.

Theorem 4.4. $U_{g}^{k}$ is a functor from the category $\mathcal{C}_{w}^{g}\left(V^{\otimes k}\right)$ of weak g-twisted $V^{\otimes k}$ modules to the category $\mathcal{C}_{w}(V)$ of weak $V$-modules such that $\left.T_{g}^{k} \circ U_{g}^{k}=i d_{\mathcal{C}_{w}^{g}(V \otimes k}\right)$ and $U_{g}^{k} \circ T_{g}^{k}=i d_{\mathcal{C}_{w}(V)}$. In particular, the categories $\mathcal{C}_{w}^{g}\left(V^{\otimes k}\right)$ and $\mathcal{C}_{w}(V)$ are isomorphic. Moreover, 
(1) The restrictions of $T_{g}^{k}$ and $U_{g}^{k}$ to the category of admissible $V$-modules $\mathcal{C}_{a}(V)$ and to the category of admissible g-twisted $V^{\otimes k}$-modules $\mathcal{C}_{a}^{g}\left(V^{\otimes k}\right)$, respectively, give category isomorphisms. In particular, $V$ is rational if and only if $V^{\otimes k}$ is g-rational.

(2) The restrictions of $T_{g}^{k}$ and $U_{g}^{k}$ to the category of ordinary $V$-modules $\mathcal{C}(V)$ and to the category of ordinary $g$-twisted $V^{\otimes k}$-modules $\mathcal{C}^{g}\left(V^{\otimes k}\right)$, respectively, give category isomorphisms.

Proof. It is trivial to verify $T_{g}^{k} \circ U_{g}^{k}=i d_{\mathcal{C}_{w}^{g}(V \otimes k)}$ and $U_{g}^{k} \circ T_{g}^{k}=i d_{\mathcal{C}_{w}(V)}$ from the definitions of the functors $T_{g}^{k}$ and $U_{g}^{k}$. Parts 1 and 2 follow from Theorem 3.9.

\section{TWISTED SECTORS FOR AN ARBITRARY $k$-CYCLE}

In this section we extend Theorem 4.4 to the category of weak $g$-twisted $V^{\otimes k}$ modules where $g$ is an arbitrary $k$-cycle. But we first recall some general results following, for example, DLM3.

Let $V$ be any vertex operator algebra, and let $g, h \in \operatorname{Aut}(V)$. There is an isomorphism from the category of weak $g$-twisted $V$-modules $\mathcal{C}_{w}^{g}(V)$ to the category of weak $h g h^{-1}$-twisted $V$-modules $\mathcal{C}_{w}^{h g h^{-1}}(V)$ given by

$$
\begin{aligned}
h: \mathcal{C}_{w}^{g}(V) & \longrightarrow \mathcal{C}_{w}^{h g h^{-1}}(V) \\
\left(M, Y_{g}\right) & \mapsto\left(M, Y_{h g h^{-1}}\right)
\end{aligned}
$$

where

$$
Y_{h g h^{-1}}(u, z)=Y_{g}(h u, z)
$$

for $u \in V$. Moreover it is clear that $\left(M, Y_{g}\right)$ is irreducible, admissible, or ordinary if and only if $\left(M, Y_{h g h^{-1}}\right)$ is irreducible, admissible, or ordinary, respectively.

Now suppose $g^{\prime}$ is an arbitrary $k$-cycle and let $g=(12 \cdots k)$. Then there exists $h \in S_{k}$ such that $g^{\prime}=h g h^{-1}$. However, this $h$ is unique only up to multiplication on the right by powers of $g$. Thus given $g^{\prime}$, we can specify $h$ uniquely by requiring that $h$ leave 1 fixed. Denote this by $h_{1}$. Then we have the following Corollary to Theorem 4.4 .

Corollary 5.1. Let $g^{\prime} \in S_{k}$ be a $k$-cycle, let $g=(12 \cdots k)$, and let $h_{1}$ be the unique element of $S_{k}$ that fixes 1 and satisfies $g^{\prime}=h_{1} g h_{1}^{-1}$. Then we have the following isomorphism of categories

$$
\begin{aligned}
T_{g^{\prime}}^{k}=h_{1} \circ T_{g}^{k}: \mathcal{C}_{w}(V) & \longrightarrow \mathcal{C}_{w}^{g^{\prime}}\left(V^{\otimes k}\right) \\
(M, Y) & \mapsto \quad\left(T_{g^{\prime}}^{k}(M), Y_{g^{\prime}}\right)=\left(M, Y_{h_{1} g h_{1}^{-1}}\right)
\end{aligned}
$$

where

$$
Y_{g^{\prime}}(v, z)=Y_{h_{1} g h_{1}^{-1}}(v, z)=Y_{g}\left(h_{1} v, z\right)
$$

for $v \in V^{\otimes k}$, and $Y_{g}$ is uniquely determined by

$$
\begin{gathered}
Y_{g}\left(u^{1}, z\right)=\bar{Y}(u, z)=Y\left(\Delta_{k}(z) u, z^{1 / k}\right) \\
Y_{g}\left(u^{j+1}, z\right)=\lim _{z^{1 / k} \rightarrow \eta^{-j} z^{1 / k}} Y_{g}\left(u^{1}, z\right)
\end{gathered}
$$

for $u \in V$. Moreover, $T_{g^{\prime}}^{k}$ preserves irreducible, admissible and ordinary objects. 
Remark 5.2. In the corollary above, we could just have easily defined $T_{g^{\prime}}^{k}$ by $T_{g^{\prime}}^{k}=$ $h_{l} \circ T_{g}^{k}$ where $h_{l}$, rather than fixing 1 , is the unique element of $S_{k}$ that takes 1 to $l$ for $l=2, \ldots, k$ and satisfies $g^{\prime}=h_{l} g h_{l}^{-1}$. Then these new $g^{\prime}$-twisted operators defined on $M$ denoted by $Y_{g^{\prime}}^{l}$ would differ from those defined in Corollary 5.1, denoted by $Y_{g^{\prime}}$, by

$$
Y_{g^{\prime}}^{l+1}\left(u^{1}, z\right)=\lim _{z^{1 / k} \rightarrow \eta^{l} z^{1 / k}} Y_{g^{\prime}}\left(u^{1}, z\right)
$$

for $l=1, \ldots, k-1$ and $u \in V$, where of course $Y_{g^{\prime}}^{l+1}(v, z)$ for $v \in V^{\otimes k}$ is determined by $Y_{g^{\prime}}^{l+1}\left(u^{j+1}, z\right)=\lim _{z^{1 / k} \rightarrow \eta^{-j} z^{1 / k}} Y_{g^{\prime}}^{l+1}\left(u^{1}, z\right)$.

\section{TWISTED SECTORS FOR ARBITRARY PERMUTATIONS}

In order to determine the various twisted module categories for an arbitrary permutation, we need to study twisted modules for tensor product vertex operator algebras in general.

For $s \in \mathbb{Z}_{+}$and $i=1, \ldots s$, let $V^{i}=\left(V^{i}, Y_{i}, \mathbf{1}_{i}, \omega_{i}\right)$ be a vertex operator algebra and $g_{i}$ an automorphism of $V^{i}$ of finite order. Then $g=g_{1} \otimes \cdots \otimes g_{s}$ is an automorphism of the tensor product vertex operator algebra $V^{1} \otimes \cdots \otimes V^{s}$ of finite order. If $W^{i}$ are weak $g_{i}$-twisted $V^{i}$-modules for $i=1, \ldots, s$ then the tensor product $W^{1} \otimes \cdots \otimes W^{s}=W$ is a weak $g$-twisted $V^{1} \otimes \cdots \otimes V^{s}$-module in the obvious way. Thus from Proposition 4.7.2 in [FH]], we have the following lemma.

Lemma 6.1. If $W^{i}$ are irreducible weak $g_{i}$-twisted $V^{i}$-modules for $i=1, \ldots, s$, then $W=W^{1} \otimes \cdots \otimes W^{s}$ is an irreducible weak $g$-twisted $V^{1} \otimes \cdots \otimes V^{s}$-module.

Proposition 6.2. The notation is the same as before. If $V^{i}$ is $g_{i}$-rational for all $i$ then $V^{1} \otimes \cdots \otimes V^{s}$ is g-rational and each irreducible $V^{1} \otimes \cdots \otimes V^{s}$-module $W$ is isomorphic to $W^{1} \otimes \cdots \otimes W^{s}$ for some irreducible $g_{i}$-twisted $V^{i}$-modules $W^{i}$.

Proof. Let $W=\oplus_{n \in \mathbb{Q}_{+}} W(n)$ be an admissible $g$-twisted $V^{1} \otimes \cdots \otimes V^{s}$-module. We need to show that $W$ is completely reducible. It is sufficient to prove that a submodule generated by any vector $w \in W(n)$ is completely reducible, and thus we can assume that $W$ is generated by $w$. Identify $V^{i}$ with the vertex operator algebra $\mathbf{1}_{1} \otimes \cdots \mathbf{1}_{i-1} \otimes V^{i} \otimes \mathbf{1}_{i+1} \otimes \cdots \otimes \mathbf{1}_{s}$. (This is "almost" a subalgebra of $V^{1} \otimes \cdots \otimes V^{s}$, failing to be a subalgebra due to the fact that $\mathbf{1}_{1} \otimes \cdots \mathbf{1}_{i-1} \otimes V^{i} \otimes \mathbf{1}_{i+1} \otimes \cdots \otimes \mathbf{1}_{s}$ has a different Virasoro element from that of $V^{1} \otimes \cdots \otimes V^{s}$.) With this identification, the submodule $W$ generated by $w$ is an admissible $g_{i}$-twisted $V^{i}$-module. By DM and [Li1], $W$ is spanned by $\left\{v_{m_{1}}^{1} \cdots v_{m_{s}}^{s} w \mid v^{i} \in V^{i}, m_{i} \in \mathbb{Q}\right\}$. Set $W^{i}$ to be the span of $\left\{v_{m} w \mid v \in V^{i}, m \in \mathbb{Q}\right\}$. Since $V^{i}$ is $g_{i}$-rational, $W^{i}$ is an admissible $g_{i}$-twisted $V^{i}$ module which is a direct sum of irreducible $g_{i}$-twisted $V^{i}$-modules. Note that the map $f$ from $W^{1} \otimes \cdots \otimes W^{s}$ to $W$ which sends $v_{m_{1}}^{1} w \otimes \cdots \otimes v_{m_{s}}^{s} w$ to $v_{m_{1}}^{1} \cdots v_{m_{s}}^{s} w$ for $v^{i} \in V^{i}, m_{i} \in \mathbb{Q}$ is a $V^{1} \otimes \cdots \otimes V^{s}$-homomorphism. By Lemma 6.1, $W^{1} \otimes \cdots \otimes W^{s}$ is completely reducible. Thus $W$ as a homomorphic image of $W^{1} \otimes \cdots \otimes W^{s}$ is completely reducible. In particular, if $W$ is irreducible then $W$ is isomorphic to a tensor product of irreducible $g_{i}$-twisted $V^{i}$-modules.

Remark 6.3. Proposition 6.2 is a generalization of a special case of a result proved in [FHL] which states that in the case $g_{i}=1$ for all $i$, any ordinary irreducible $V^{1} \otimes \cdots \otimes V^{s}$-module $W$ on whose lowest weight space the operators $L_{i}(0)$ have only rational eigenvalues is isomorphic to the tensor product of some irreducible 
$V^{i}$-modules. In our case $L_{i}(0)$ is the component operator of $Y_{i}\left(\omega_{i}, z\right)$ of weight 0 , the $g_{i}$ are general automorphisms of $V^{i}$ of finite order, but we have restricted to the case where each $V^{i}$ is $g_{i}$-rational.

Let $k$ be a fixed positive integer, and let $g \in S_{k}$. Then $g$ can be written as a product of disjoint cycles $g=g_{1} \cdots g_{p}$ where the order of $g_{i}$ is $k_{i}$ such that $\sum_{i} k_{i}=$ $k$. (Note that we are including 1-cycles.) Furthermore, there exists $h \in S_{k}$ satisfying $g=h g_{1}^{\prime} \cdots g_{p}^{\prime} h^{-1}$ such that $g_{i}^{\prime}$ is a $k_{i}$-cycle which permutes the numbers $\left(\sum_{j=1}^{i-1} k_{j}\right)+$ $1,\left(\sum_{j=1}^{i-1} k_{j}\right)+2, \ldots, \sum_{j=1}^{i} k_{j}$. In Sections 3,4 and 5 we have determined various $g_{i}$-twisted $V^{\otimes k_{i}}$-module categories in terms of corresponding $V$-module categories via the functor $T_{g_{i}}^{k_{i}}$ for $g_{i}$ a $k_{i}$-cycle. Thus we have the following:

Theorem 6.4. Let $W_{i}$ be a weak $V$-module for $i=1, \ldots, p$. Given $g \in S_{k}$ and $a$ decomposition $g=h g_{1}^{\prime} \cdots g_{p}^{\prime} h^{-1}$ as above, $h \circ\left(T_{g_{1}^{\prime}}^{k_{1}}\left(W_{1}\right) \otimes \cdots \otimes T_{g_{p}^{\prime}}^{k_{p}}\left(W_{p}\right)\right)$ is a weak $g$-twisted $V^{\otimes k}$-module. Moreover,

(1) If each $W_{i}$ is irreducible then $h \circ\left(T_{g_{1}^{\prime}}^{k_{1}}\left(W_{1}\right) \otimes \cdots \otimes T_{g_{p}^{\prime}}^{k_{p}}\left(W_{p}\right)\right)$ is irreducible.

(2) If each $W_{i}$ is an admissible (resp., ordinary) $V$-module, then $h \circ\left(T_{g_{1}^{\prime}}^{k_{1}}\left(W_{1}\right) \otimes\right.$ $\cdots \otimes T_{g_{p}^{\prime}}^{k_{p}}\left(W_{p}\right)$ ) is an admissible (resp., ordinary) g-twisted $V^{\otimes k}$-module.

(3) If $V$ is a rational vertex operator algebra, then $V^{\otimes k}$ is g-rational and all irreducible g-twisted modules are given by $h \circ\left(T_{g_{1}^{\prime}}^{k_{1}}\left(W_{1}\right) \otimes \cdots \otimes T_{g_{p}^{\prime}}^{k_{p}}\left(W_{p}\right)\right)$ where $W_{i}$ are irreducible $V$-modules.

Proof. Parts (1) and (2) follow from Corollary 5.1 and Lemma 6.1. Part (3) follows from Corollary 5.1 and Proposition 6.2.

Remark 6.5. Note that in the Theorem above, we have not specified a unique decomposition $g=h g_{1}^{\prime} \cdots g_{p}^{\prime} h^{-1}$ but rather have given the functor $h \circ\left(T_{g_{1}^{\prime}}^{k_{1}} \otimes \cdots \otimes T_{g_{p}^{\prime}}^{k_{p}}\right)$ for a given such (non-unique) decomposition $g=h g_{1}^{\prime} \cdots g_{p}^{\prime} h^{-1}$. However, for any decomposition $g=h g_{1}^{\prime} \cdots g_{p}^{\prime} h^{-1}$, the resulting $g$-twisted $V^{\otimes k}$-module $h \circ\left(T_{g_{1}^{\prime}}^{k_{1}}\left(W_{1}\right) \otimes\right.$ $\left.\cdots \otimes T_{g_{p}^{\prime}}^{k_{p}}\left(W_{p}\right)\right)$ is isomorphic to that obtained from any other decomposition. Similar to the symmetries discussed in Remarks 3.11 and 5.2 above, the isomorphism consists of transformations $\lim _{z^{1 / k_{i}} \rightarrow \eta_{k_{i}}^{-j_{i}} z^{1 / k_{i}}}$ of each $i$-th tensor factor in the $g$ twisted vertex operator where $\eta_{k_{i}}=e^{-2 \pi i / k_{i}}$ and $j_{i}=1, \ldots, k_{i}-1$.

\section{Twisted SECTORS FOR THE PROdUCT OF A PERMUtATION WITH AN AUTOMORPHISM OF $V$}

In this section we give a slight generalization of our previous results to a broader class of automorphisms. Note that $\operatorname{Aut}(V)$ acts on $V^{\otimes k}$ diagonally and this action commutes with the action of $S_{k}$. Let $\gamma \in \operatorname{Aut}(V)$ and $g \in S_{k}$. In this section we determine various $\gamma g$-twisted $V^{\otimes k}$-module categories. (Although the diagonal action of $\gamma$ on $V^{\otimes k}$ is more appropriately denoted by $\gamma^{\otimes k}$, to simplify notation, we will write this as $\gamma$ with the diagonal action being understood.)

First, we assume that $g=(12 \cdots k)$ and show that suitable variants of all the arguments and results in Sections 3 and 4 remain valid in for the case of $\gamma g$-twisted $V^{\otimes k}$-module categories. Then we generalize the permutation $g$ as we did in Sections 5 and 6 and show that again suitable variants of our arguments remain valid. 
We proceed to formulate these results. Let $g=(12 \cdots k)$. For each positive integer $n$, set $\eta_{n}=e^{-2 \pi i / n}$. Then $\eta_{n / m}=\eta_{n}^{m}$. Let $o(\gamma)=l$, and set $o(\gamma)=l=s d$ where $d$ is the greatest common divisor of $l$ and $k$. Furthermore, if we let $o(\gamma g)=n$, then $o(\gamma g)=n=s k$. Let $(W, Y)$ be a weak $\gamma g$-twisted $V^{\otimes k}$-module. Then the analogue of equation (3.4) is

$$
\begin{gathered}
z_{0}^{-1} \delta\left(\frac{z_{1}-z_{2}}{z_{0}}\right) Y\left(u^{1}, z_{1}\right) Y\left(v^{1}, z_{2}\right)-z_{0}^{-1} \delta\left(\frac{z_{2}-z_{1}}{-z_{0}}\right) Y\left(v^{1}, z_{2}\right) Y\left(u^{1}, z_{1}\right) \\
=\frac{1}{n} z_{2}^{-1} \sum_{j=0}^{n-1} \delta\left(\eta_{n}^{j} \frac{\left(z_{1}-z_{0}\right)^{1 / n}}{z_{2}^{1 / n}}\right) Y\left(Y\left(\gamma^{j} g^{j} u^{1}, z_{0}\right) v^{1}, z_{2}\right)
\end{gathered}
$$

for $u, v \in V$. Let $u$ be an eigenvector for $\gamma$ with eigenvalue $\eta_{l}^{r}$ for some $r=1, \ldots, l$. Then $\gamma^{j} g^{j} u^{1}=\eta_{l}^{r j} u^{j+1}$ where $u^{j+k}=u^{j}$. Thus similar to the situation in Section $3, Y\left(\gamma^{j} g^{j} u^{1}, z_{0}\right) v^{1}$ only involves nonnegative integer powers of $z_{0}$ if $j \neq 0(\bmod k)$, and

$$
\begin{aligned}
& {\left[Y\left(u^{1}, z_{1}\right), Y\left(v^{1}, z_{2}\right)\right]=} \\
&= \sum_{q=0}^{s-1} \operatorname{Res}_{z_{0}} \frac{1}{n} z_{2}^{-1} \delta\left(\eta_{n}^{k q} \frac{\left(z_{1}-z_{0}\right)^{1 / n}}{z_{2}^{1 / n}}\right) Y\left(Y\left(\eta_{l}^{r k q} u^{1}, z_{0}\right) v^{1}, z_{2}\right) \\
&= \sum_{q=0}^{s-1} \operatorname{Res}_{z_{0}} \frac{1}{n} z_{2}^{-1} \delta\left(\eta_{s}^{q} \frac{\left(z_{1}-z_{0}\right)^{1 / n}}{z_{2}^{1 / n}}\right) \eta_{s}^{r q k / d} Y\left(Y\left(u^{1}, z_{0}\right) v^{1}, z_{2}\right) \\
&= \sum_{q=0}^{s-1} \sum_{p=0}^{n-1} \operatorname{Res}_{z_{0}} \frac{1}{n} z_{2}^{-1} \eta_{s}^{q p}\left(\frac{z_{1}-z_{0}}{z_{2}}\right)^{p / n} \delta\left(\frac{z_{1}-z_{0}}{z_{2}}\right) \eta_{s}^{r q k / d} \\
&= \sum_{q=0}^{s-1} \sum_{t=1}^{k} \operatorname{Res}_{z_{0}} \frac{1}{s k} z_{2}^{-1}\left(\frac{z_{1}-z_{0}}{z_{2}}\right)^{(t s-r k / d) / n} \delta\left(\frac{z_{1}-z_{0}}{z_{2}}\right) \\
&= \sum_{t=1}^{k} \operatorname{Res}_{z_{0}} \frac{1}{k} z_{2}^{-1}\left(\frac{\left.\left.z_{1}-z_{0}^{1}, z_{0}\right) v^{1}, z_{2}\right)}{z_{2}}\right)^{t / k}\left(\frac{z_{1}-z_{0}}{z_{2}}\right)^{-r / l} \delta\left(\frac{z_{1}-z_{0}}{z_{2}}\right) \\
&(7.2)=\left.\operatorname{Res}_{z_{0}} \frac{1}{k} z_{2}^{-1}\left(\frac{z_{1}-z_{0}}{z_{2}}\right)^{-r / l} \delta\left(\frac{\left(z_{1}-z_{0}\right)^{1 / k}}{z_{2}^{1 / k}}\right) v^{1}, z_{2}\right) \\
&\left.Y\left(u^{1}, z_{0}\right) v^{1}, z_{2}\right) \\
&\left.Y\left(u^{1}, z_{0}\right) v^{1}, z_{2}\right) .
\end{aligned}
$$

As in Section 3 this shows that for $u \in V$ the component operators of $Y\left(u^{1}, z\right)$ on $W$ form a Lie algebra.

Let $(M, Y)$ be a weak $\gamma^{k}$-twisted $V$-module. Set $\bar{Y}(u, z)=Y\left(\Delta_{k}(z) u, z^{1 / k}\right)$.

Lemma 7.1. The assertion of Lemma 3.9 holds in the present setting: for $u \in V$

$$
\bar{Y}(L(-1) u, z)=\frac{d}{d z} \bar{Y}(u, z)
$$

Proof. The steps of the proof for Lemma 3.2 still hold in the present setting for $(M, Y)$ a $\gamma^{k}$-twisted $V$-module rather than just a $V$-module.

The analogue of Lemma 3.3 is 
Lemma 7.2. For $u, v \in V$ such that $\gamma u=\eta_{l}^{r} u$, we have

$$
\left[\bar{Y}\left(u, z_{1}\right), \bar{Y}\left(v, z_{2}\right)\right]=\operatorname{Res}_{z_{0}} \frac{1}{k} z_{2}^{-1}\left(\frac{z_{1}-z_{0}}{z_{2}}\right)^{-r / l} \delta\left(\frac{\left(z_{1}-z_{0}\right)^{1 / k}}{z_{2}^{1 / k}}\right) \bar{Y}\left(Y\left(u, z_{0}\right) v, z_{2}\right) .
$$

Proof. From the twisted Jacobi identity, we have

$$
\left[Y\left(u, z_{1}\right), Y\left(v, z_{2}\right)\right]=\operatorname{Res}_{x} z_{2}^{-1}\left(\frac{z_{1}-x}{z_{2}}\right)^{-r / l} \delta\left(\frac{z_{1}-x}{z_{2}}\right) Y\left(Y(u, x) v, z_{2}\right)
$$

for $u \in V$ such that $\gamma u=\eta_{l}^{r} u$. Following the steps of the proof of Lemma 3.3 using the commutator formula (7.4) instead of (3.10) gives (7.3).

For $u \in V$ such that $\gamma u=\eta_{l}^{r}$ define operators $Y_{\gamma g}$ on $V^{\otimes k}$ by

$$
\begin{aligned}
& Y_{\gamma g}\left(u^{1}, z\right)=\bar{Y}(u, z)=Y\left(\Delta_{k}(z) u, z^{1 / k}\right) \\
& \eta_{l}^{r j} Y_{\gamma g}\left(u^{j+1}, z\right)=\lim _{z^{1 / n} \rightarrow \eta_{n}^{-j} z^{1 / n}} Y\left(u^{1}, z\right)
\end{aligned}
$$

for $j=0, \ldots, k-1$. Then as before, the operators $Y_{\gamma g}\left(u^{j}, z\right)$ for $u \in V$ and $j=1, \ldots, k$ are mutually local and generate a local system $A$ in the sense of [Li2. Let $\sigma$ be a map from $A$ to $A$ such that $\sigma Y_{\gamma g}\left(u^{j}, z\right)=\eta_{l}^{-r} Y_{\gamma g}\left(u^{j+1}, z\right)$ for $u \in V$ satisfying $\gamma u=\eta_{l}^{r} u$, and for $j=1, \ldots, k$. Again $A$ has the structure of a vertex algebra $\left(A, Y_{A}\right)$ and $\sigma$ extends to an automorphism of $A$ of order $n$ such that $M$ is naturally a weak $\sigma$-twisted $A$-module in the sense that $Y\left(\alpha(z), z_{1}\right)=\alpha\left(z_{1}\right)$ for $\alpha(z) \in A$.

Lemma 7.3. The assertions of Lemmas 3.6, 3.7 and 3.8 hold for $Y_{\gamma g}$ and the corresponding local system.

Proof. The steps of the proofs of Lemmas 3.6, 3.7 and 3.8 remain valid in the present setting.

Let $(M, Y)$ be a weak $\gamma^{k}$-twisted $V$-module. Define

$$
T_{\gamma g}^{k}(M, Y)=\left(T_{\gamma g}^{k}(M), Y_{\gamma g}\right)=\left(M, Y_{\gamma g}\right) .
$$

That is $T_{\gamma g}^{k}(M, Y)$ is $M$ as the underlying vector space and the vertex operator $Y_{\gamma g}$ is given by $(7.5)$ and $(7.6)$.

Now repeating the proof of Theorem 3.9 under the present circumstances, we have the following result which is a generalization of Theorem 3.9.

Theorem 7.4. $\left(T_{\gamma g}^{k}(M), Y_{\gamma g}\right)$ is a weak $\gamma g$-twisted $V^{\otimes k}$-module such that $T_{\gamma g}^{k}(M)=$ $M$ and $Y_{\gamma g}$, defined by (7.5) and (7.0), is the linear map from $V^{\otimes n}$ to

$$
\text { (End } \left.T_{\gamma g}^{k}(M)\right)\left[\left[z^{1 / n}, z^{-1 / n}\right]\right]
$$

defining the twisted module structure where $n=o(\gamma g)$. Moreover,

(1) $(M, Y)$ is an irreducible weak $\gamma^{k}$-twisted $V$-module if and only if $\left(T_{\gamma g}^{k}(M), Y_{\gamma g}\right)$ is an irreducible weak $\gamma g$-twisted $V^{\otimes k}$-module.

(2) $M$ is an admissible $\gamma^{k}$-twisted $V$-module if and only if $T_{\gamma g}^{k}(M)$ is an admissible $\gamma g$-twisted $V^{\otimes k}$-module.

(3) $M$ is an ordinary $\gamma^{k}$-twisted $V$-module if and only if $T_{\gamma g}^{k}(M)$ is an ordinary $\gamma g$-twisted $V^{\otimes k}$-module. 
Let

$$
\begin{aligned}
T_{\gamma g}^{k}: \mathcal{C}_{w}^{\gamma^{k}}(V) & \longrightarrow \mathcal{C}_{w}^{\gamma g}\left(V^{\otimes k}\right) \\
(M, Y) & \mapsto\left(T_{\gamma g}^{k}(M), Y_{\gamma g}\right)=\left(M, Y_{\gamma g}\right) \\
f & \mapsto T_{\gamma g}^{k}(f)=f
\end{aligned}
$$

for $(M, Y)$ an object and $f$ a morphism in $\mathcal{C}_{w}^{\gamma^{k}}(V)$. We have the following corollary to Theorem 7.4.

Corollary 7.5. $T_{\gamma g}^{k}$ is a functor from the category $\mathcal{C}_{w}^{\gamma^{k}}(V)$ to the category $\mathcal{C}_{w}^{\gamma g}\left(V^{\otimes k}\right)$ such that: (1) $T_{\gamma g}^{k}$ preserves irreducible objects; (2) The restrictions of $T_{\gamma g}^{k}$ to $\mathcal{C}_{a}^{\gamma^{k}}(V)$ and $\mathcal{C}^{\gamma^{k}}(V)$ are functors from $\mathcal{C}_{a}^{\gamma^{k}}(V)$ and $\mathcal{C}^{\gamma^{k}}(V)$ to $\mathcal{C}_{a}^{\gamma g}\left(V^{\otimes k}\right)$ and $\mathcal{C}^{\gamma g}\left(V^{\otimes k}\right)$, respectively.

Note that if $\gamma$ is the identity, Theorem 7.4 and Corollary 7.5 reduce to Theorem 3.9 and Corollary 3.10, respectively.

Now let $M=\left(M, Y_{\gamma g}\right)$ be a weak $\gamma g$-twisted $V^{\otimes k}$-module. In analogy to Section 4, we set $U_{\gamma g}^{k}(M)=M$ and define $Y_{U}(u, z)=Y_{\gamma g}\left(\left(\Delta_{k}\left(z^{k}\right)^{-1} u\right)^{1}, z^{k}\right)$ for $u \in V$ to be the unique power series obtained by letting $\left(z^{k}\right)^{1 / k}=z$. Lemma 4.1 remains valid in this case and Lemma 4.2 is modified as follows:

Lemma 7.6. Let $u, v \in V$ such that $\gamma u=\eta_{l}^{r}$. Then on $U_{\gamma g}^{k}(M)$,

$$
\left[Y_{U}\left(u, z_{1}\right), Y_{U}\left(v, z_{2}\right)\right]=\operatorname{Res}_{z_{0}} z_{2}^{-1}\left(\frac{z_{1}-z_{0}}{z_{2}}\right)^{-r k / l} \delta\left(\frac{z_{1}-z_{0}}{z_{2}}\right) Y_{U}\left(Y\left(u, z_{0}\right) v, z_{2}\right)
$$

Proof. The $\gamma g$-twisted vertex operators satisfy

$$
\begin{aligned}
& {\left[Y_{\gamma g}\left(u^{1}, z_{1}\right), Y_{\gamma g}\left(v^{1}, z_{2}\right)\right]} \\
& \quad=\operatorname{Res}_{z_{0}} \frac{1}{k} z_{2}^{-1}\left(\frac{z_{1}-z_{0}}{z_{2}}\right)^{-r / l} \delta\left(\frac{\left(z_{1}-z_{0}\right)^{1 / k}}{z_{2}^{1 / k}}\right) Y_{\gamma g}\left(Y\left(u^{1}, z_{0}\right) v^{1}, z_{2}\right) .
\end{aligned}
$$

Following the steps for the proof of Lemma 4.2 using (7.8) instead of (4.2) gives (7.7).

Repeating the proof of Theorem 4.3 in the present setting gives:

Theorem 7.7. With the notations as above, $\left(U_{\gamma g}^{k}(M), Y_{U}\right)$ is a weak $\gamma^{k}$-twisted V-module.

Thus we have the following analogue of Theorem 4.4 .

Theorem 7.8. $U_{\gamma g}^{k}$ is a functor from the category $\mathcal{C}_{w}^{\gamma g}\left(V^{\otimes k}\right)$ of weak $\gamma g$-twisted $V^{\otimes k}$-modules to the category $\mathcal{C}_{w}^{\gamma^{k}}(V)$ of weak $\gamma^{k}$-twisted $V$-modules such that $T_{\gamma g}^{k} \circ$ $U_{\gamma g}^{k}=i d_{\mathcal{C}_{w}^{\gamma g}\left(V^{\otimes k}\right)}$ and $U_{\gamma g}^{k} \circ T_{\gamma g}^{k}=i d_{\mathcal{C}_{w}^{\gamma^{k}}(V)}$. In particular, the categories $\mathcal{C}_{w}^{\gamma g}\left(V^{\otimes k}\right)$ and $\mathcal{C}_{w}^{\gamma^{k}}(V)$ are isomorphic. Moreover,

(1) The restrictions of $T_{\gamma g}^{k}$ and $U_{\gamma g}^{k}$ to the category of admissible $V$-modules $\mathcal{C}_{a}^{\gamma^{k}}(V)$ and to the category of admissible $\gamma g$-twisted $V^{\otimes k}$-modules $\mathcal{C}_{a}^{\gamma g}\left(V^{\otimes k}\right)$, respectively, give category isomorphisms. In particular, $V$ is $\gamma^{k}$-rational if and only if $V^{\otimes k}$ is $\gamma g$-rational. 
(2) The restrictions of $T_{\gamma g}^{k}$ and $U_{\gamma g}^{k}$ to the category of ordinary $V$-modules $\mathcal{C}^{\gamma^{k}}(V)$ and to the category of ordinary $\gamma g$-twisted $V^{\otimes k}$-modules $\mathcal{C}^{\gamma g}\left(V^{\otimes k}\right)$, respectively, give category isomorphisms.

Recalling the conjugation functor $h$ from Section 5, and again noting that the diagonal action of $\gamma$ on $V^{\otimes k}$ commutes with that of the symmetric group so that $\gamma h g h^{-1}=h \gamma g h^{-1}$, we have the following corollary to Theorem 7.8.

Corollary 7.9. Let $g^{\prime} \in S_{k}$ be a $k$-cycle, let $g=(12 \cdots k)$ and let $h_{1}$ be the unique element of $S_{k}$ that fixes 1 and satisfies $g^{\prime}=h_{1} g h_{1}^{-1}$. Then we have the following isomorphism of categories

$$
\begin{aligned}
T_{\gamma g^{\prime}}^{k}=h_{1} \circ T_{\gamma g}^{k}: \mathcal{C}_{w}^{\gamma^{k}}(V) & \longrightarrow \mathcal{C}_{w}^{\gamma g^{\prime}}\left(V^{\otimes k}\right) \\
(M, Y) & \mapsto \quad\left(T_{\gamma g^{\prime}}^{k}(M), Y_{\gamma g^{\prime}}\right)=\left(M, Y_{h_{1} \gamma g h_{1}^{-1}}\right)
\end{aligned}
$$

where

$$
Y_{h_{1} \gamma g h_{1}^{-1}}(v, z)=Y_{\gamma g}\left(h_{1} v, z\right)
$$

and $Y_{\gamma g}$ is uniquely determined by (7.5) and (7.0.). Moreover, $T_{\gamma g^{\prime}}^{k}$ preserves irreducible, admissible and ordinary objects.

Finally we deal with the case $\gamma g$ when $g$ is an arbitrary permutation in $S_{k}$. As in Section 6 we write $g$ as a product of disjoint cycles $g=h g_{1}^{\prime} \cdots g_{p}^{\prime} h^{-1}$ where the order of $g_{i}^{\prime}$ is $k_{i}$ such that $\sum_{i} k_{i}=k$ and where $g_{i}$ permutes the numbers $\left(\sum_{j=1}^{i-1} k_{j}\right)+$ $1,\left(\sum_{j=1}^{i-1} k_{j}\right)+2, \ldots, \sum_{j=1}^{i} k_{j}$. The following theorem generalizes Theorem 6.4.

Theorem 7.10. Let $W_{i}$ be a weak $\gamma^{k_{i}}$-twisted $V$-module for $i=1, \ldots, p$. Given $g \in$ $S_{k}$ and a decomposition $g=h g_{1}^{\prime} \cdots g_{p}^{\prime} h^{-1}$ as above, $h \circ\left(T_{\gamma g_{1}}^{k_{1}}\left(W_{1}\right) \otimes \cdots \otimes T_{\gamma g_{p}}^{k_{p}}\left(W_{p}\right)\right)$ is a weak $\gamma g$-twisted $V^{\otimes k}$-module. Moreover,

(1) If each $W_{i}$ is irreducible, then $h \circ\left(T_{\gamma g_{1}}^{k_{1}}\left(W_{1}\right) \otimes \cdots \otimes T_{\gamma g_{p}}^{k_{p}}\left(W_{p}\right)\right)$ is irreducible.

(2) If each $W_{i}$ is an admissible (resp., ordinary) $V$-module, then $h \circ\left(T_{\gamma g_{1}}^{k_{1}}\left(W_{1}\right) \otimes\right.$ $\left.\cdots \otimes T_{\gamma g_{p}}^{k_{p}}\left(W_{p}\right)\right)$ is an admissible (resp., ordinary) $\gamma g$-twisted $V^{\otimes k}$-module.

(3) If $V$ is a $\gamma^{k_{i}}$-rational vertex operator algebra for $i=1, \ldots, p$, then $V^{\otimes k}$ is $\gamma g$-rational and all irreducible $\gamma g$-twisted modules are given by $h \circ\left(T_{\gamma g_{1}}^{k_{1}}\left(W_{1}\right) \otimes \cdots \otimes\right.$ $\left.T_{\gamma g_{p}}^{k_{p}}\left(W_{p}\right)\right)$ where $W_{i}$ are irreducible $\gamma^{k_{i}}$-twisted $V$-modules.

Of course the analogous symmetries discussed in Remark 5.2 and Remark 6.5 hold for Corollary 7.9 and Theorem 7.10, respectively.

\section{REFERENCES}

[Ba] P. Bantay, Characters and modular properties of permutation orbifolds, hep-th/9708120.

[Bo] R. E. Borcherds, Vertex algebras, Kac-Moody algebras, and the Monster, Proc. Natl. Acad. Sci. USA 83 (1986), 3068-3071.

[BHS] L. Borisov, M.B. Halpern and C. Schweigert, Systematic approach to cyclic orbifolds, hep-th/9701061.

[DHVW1] L.J. Dixon, J.A. Harvey, C. Vafa, and E. Witten, Strings on orbifolds, Nucl. Phys. B $261(1985) 678$.

[DHVW2] L.J. Dixon, J.A. Harvey, C. Vafa, and E. Witten, Strings on orbifolds, II, Nucl. Phys. B 274 (1986) 285.

[DVVV] R. Dijkgraaf, C. Vafa, E. Verlinde and H. Verlinde, The operator algebra of orbifold models, Comm. Math. Phys. 123 (1989), 485-526. 
[DMVV] R. Dijkgraaf, G. Moore, E. Verlinde and H. Verlinde, Elliptic genera of symmetric products and second quantized strings, Comm. Math. Phys. 185 (1997), 197.

[D] C. Dong, Twisted modules for vertex algebras associated with even lattices, J. Algebra 165 (1994), 90-112.

[DL1] C. Dong and J. Lepowsky, Generalized Vertex Algebras and Relative Vertex Operators, Progress in Math. Vol. 112, Birkhäuser, Boston, 1993.

[DL2] C. Dong and J. Lepowsky, The algebraic structure of relative twisted vertex operators, J. Pure Appl. Algebra 110 (1996), no. 3, 259-295.

[DLM1] C. Dong, H. Li and G. Mason, Regularity of rational vertex operator algebras, Adv. in Math. 132 (1997), 148-166.

[DLM2] C. Dong, H. Li and G. Mason, Twisted representations of vertex operator algebras, Math. Ann. 310 (1998), 571-600.

[DLM3] C.Dong. H. Li and G. Mason, Modular invariance of trace functions in orbifold theory, q-alg/9703016.

[DM] C. Dong and G. Mason, Nonabelian orbifolds and boson-fermion correspondence, Comm. Math. Phys. 163 (1994), 523-559.

[FFR] A. J. Feingold, I. B. Frenkel and J. F. X. Ries, Spinor Construction of Vertex Operator Algebras, Triality and $E_{8}^{(1)}$, Contemp. Math. 121, Amer. Math. Soc., Providence, 1991.

[FHL] I. B. Frenkel, Y. Huang and J. Lepowsky, On axiomatic approaches to vertex operator algebras and modules, Memoirs American Math. Soc. 104, 1993.

[FLM1] I. B. Frenkel, J. Lepowsky and A. Meurman, A natural representation of the FischerGriess Monster with the modular function $J$ as character, Proc. Natl. Acad. Sci. USA 81 (1984), 3256-3260.

[FLM2] I. B. Frenkel, J. Lepowsky and A. Meurman, Vertex operator calculus, in: Mathematical Aspects of String Theory, Proc. 1986 Conference, San Diego. ed. by S.-T. Yau, World Scientific, Singapore, 1987, 150-188.

[FLM3] I. B. Frenkel, J. Lepowsky and A. Meurman, Vertex Operator Algebras and the Monster, Pure and Applied Math., Vol. 134, Academic Press, 1988.

[FKS] J. Fuchs, A. Klemm and M.G. Schmidt, Ann. Phys. 214 (1992), 221.

[GKO] P. Goddard, A. Kent and D. Olive, Unitary representations of the Virasoro Algebra and super-Virasoro algebras Comm. Math. Phys. 103 (1986), 105-119.

[H] Y. Huang, Two Dimensional Conformal Geometry and Vertex Operator Algebras, Progress in Math. Vol. 148, Birkhäuser, Boston, 1997.

[KS] A. Klemm and M.G. Schmidt, Phys. Lett. B245 (1990), 53.

[Le1] J. Lepowsky, Calculus of twisted vertex operators, Proc. Nat. Acad. Sci. USA 82 (1985), no. 24, 8295-8299.

[Le2] J. Lepowsky, Perspectives on vertex operators and the Monster, in: Proc. 1987 Symposium on the Mathematical Heritage of Hermann Weyl, Duke Univ., Proc. Symp. Pure Math., American Math. Soc., 48 (1988).

[Li1] H. Li, An approach to tensor product theory for representations of a vertex operator algebra, Ph.D. thesis, Rutgers University, 1994.

[Li2] H. Li, Local systems of twisted vertex operators, vertex superalgebras and twisted modules, Contemporary Math. 193 (1996), 203-236.

[Z] Y.-C. Zhu, Modular invariance of characters of vertex operator algebras, J. Amer. Math. Soc. 9 (1996), 237-302.

Department of Mathematics, University of California, Santa Cruz, CA 95064

E-mail address: kbarron@math.ucsc.edu

Department of Mathematics, University of California, Santa Cruz, CA 95064

E-mail address: dong@math.ucsc.edu

Department of Mathematics, University of California, Santa Cruz, CA 95064

E-mail address: gem@math.ucsc.edu 